\title{
capacité portante d'une fondation de grande longueur sur un sol cohérent non homogène
}

\author{
par \\ A. Bottero \\ Maître-Assistant I. U.T. 1 Grenoble \\ Institut de Recherches Interdisciplinaires \\ de Géologie et de Mécanique
}

\section{Introduction}

La capacité portante des fondations reposant sur des sols non homogènes a été l'objet de nombreuses études théoriques et expérimentales, les premières s'appuyant fréquemment sur l'hypothèse d'un schéma de rupture imposé (cercles, spirales logarithmiques...).

L'utilisation de méthodes numériques basées sur la discrétisation du milieu en éléments finis a permis de s'affranchir des difficultés afférentes aux conditions, aux limites, de ne faire aucune hypothèse sur le schéma de rupture, de tenir compte de la nature des interfaces et d'obtenir des solutions statique et cinématique dont le caractère licite peut être contrôlé facilement. La conception, la mise en œuvre des programmes ayant servi à cette double approche sont explicitées dans les références [7] et [8], les premières applications sont détaillées en [3] et [7] : nous ne rappelons ici que les éléments indispensables à la compréhension des problèmes traités.

2 Approches statique et cinématique de la pression limite sous une semelle reposant sur un sol constitué de deux couches cohérentes superposées et limité inférieurement par un substratum indéformable

\subsection{Conditions aux limites et hypothèses de} calcul

On considère en déformation plane, une fondation rigide de largeur $B$ reposant sur un sol rigide-plastique obéissant au critère de plasticité de TRESCA et constitué de deux couches horizontales d'épaisseurs $h_{1}$ et $h_{2}$ et de cohésions $c_{1}$ et $c_{2}$ (fig. 1). A la profondeur $\mathrm{h}$, le sol est limité par un substratum indéformable. L'interface fondation-couche 1 est de type rugueux standard de TRESCA ainsi que l'interface couche 2-substratum, soit :

$$
\left|\tau_{n} t\right| \leqslant C \text { et }\left[U_{t}\right] \geqslant 0, \quad\left[U_{n}\right]=0 \text {; }
$$

$\left[U_{t}\right]$ désignant la discontinuité cinématique tangentielle éventuelle et $\left[\mathrm{U}_{n}\right]$ la discontinuité normale de la vitesse. La surface du sol est non chargée. Les deux couches sont collées ou peuvent présenter une ligne de discontinuité cinématique au niveau de l'intercouche.

2.2 Approche statique de la pression limite exercée par la fondation sur le sol

La méthode statique de l'analyse limite consiste ici à rechercher le maximum de :

$$
F=\int_{A A^{*}} \sigma x d y
$$

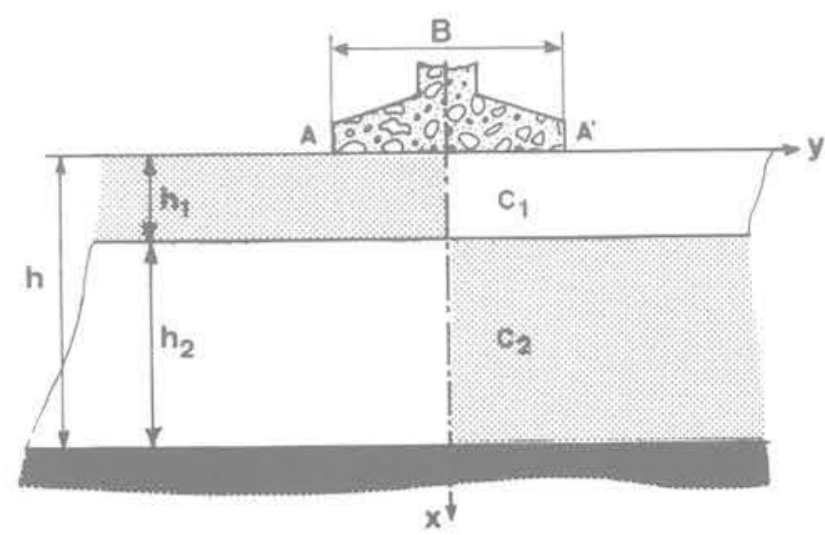

Fig. 1 Fondation sur bi-couche cohérent limité par un substratum indéformable 
parmi l'ensemble des champs de contraintes licites autorisés par une discrétisation en éléments finis triangulaires sur lesquels la forme du tenseur contrainte :

$$
\sigma_{i j}=a_{i j}+b_{i j} x+c_{i j} y
$$

permet d'exprimer linéairement l'ensemble des conditions d'admissibilité statique suivant les composantes nodales des tenseurs contraintes $\left(\{\sigma\}^{\circ}\right.$ pour un triangle (e)). Pour éviter la non-linéarité introduite par les conditions d'admissibilité plastique $f\left(\sigma_{i 1}\right) \leqslant 0$, le critère de TRESCA est remplacé par un critère linéarisé par l'intérieur [8]. Des discontinuités en contraintes sont autorisées au passage d'un élément à un autre afin d'élargir la classe des champs $q$ que la méthode permet d'explorer. Enfin des conditions de prolongement des champs de contraintes détaillées dans [7] sont imposées dans certaines directions (fig. 2 b) afin de garantir la validité mécanique des solutions obtenues, au-delà des frontières du modèle.

Le vecteur $\{\sigma\}$ désignant l'ensemble des composantes des tenseurs contraintes $\{\sigma\}^{\mathrm{e}}$ nodaux du modèle, la borne statique de $p_{\ell}=F / B$ s'obtient en résolvant le problème d'optimisation linéaire :

$$
\begin{aligned}
\operatorname{Max} F & =\int_{A A^{\prime}} \sigma_{x} d y \\
A\{\sigma\} & \leqslant\{a\}
\end{aligned}
$$

A et $\{a\}$ provenant des conditions d'admissibilité statique et plastique imposées à $\underline{\sigma}$.

\subsection{Approche cinématique de la pression limite}

La méthode cinématique consiste à chercher le minimum de la fonctionnelle :

$P(\dot{\varepsilon})=\sum_{i=1}^{N} C_{i} \int_{S_{1}} \sqrt{2 \dot{\varepsilon}_{k \varepsilon} \dot{\varepsilon}_{k \ell}} d S_{i}+\sum_{d=1}^{D} C^{d}\left|\left[U_{t}\right]\right| \cdot \ell^{d}$

dans laquelle $\dot{\varepsilon}$ représente un champ de vitesses de déformation licite dérivant, au sens des petites déformations, d'un champ de vitesses de déplacement à variation linéaire :

$$
\mathrm{U}_{1}=\mathrm{a}_{\mathrm{i}}+\mathrm{b}_{\mathrm{i}} \mathrm{x}+\mathrm{c}_{\mathrm{i}} \mathrm{y}
$$

défini sur chacun des $\mathrm{N}$ éléments triangulaires et des $\mathrm{D}$ discontinuités cinématiques d'un modèle éléments finis tel que celui de la figure 3 .

L'expression du théorème des puissances virtuelles

$$
\begin{aligned}
\mathrm{P}(\dot{\varepsilon}) & =F_{x} V_{0}+\gamma_{x} \dot{q}_{y} \\
\dot{q}_{x} & =\sum_{i=1}^{N} \int_{S_{1}} U_{x} \cdot d S_{i}=0
\end{aligned}
$$

conduit à un majorant de la force $\mathrm{F}$ d'enfoncement vertical à la vitesse $\mathrm{V}_{0}[10]$

L'ensemble des conditions d'admissibilité cinématiques et plastiques s'expriment linéairement suivant les vitesses nodales $\left(\{U\}^{e}\right.$ pour un élément (e)). Pour se ramener globalement à un problème d'optimisation linéaire on utilise un critère de TRESCA linéarisé par l'extérieur [8], ce qui conduit à un problème cinématique global :

$\operatorname{Min} P(\lambda, u)=2 \sum_{i=1}^{N}\left(C_{i} S_{i} \sum_{j=1}^{m} \lambda_{i j}\right)+\sum_{d=1}^{D}\left|\left[U_{t}\right]\right| \ell^{d}$ $B\left\{\begin{array}{l}\lambda \\ u\end{array}\right\}=0$

avec : $\lambda_{i j}=$ coefficients issus de la dérivation du critère de TRESCA linéarisé; (a)

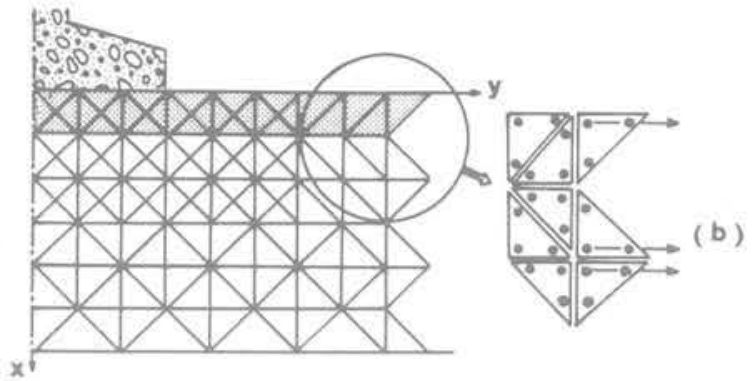

Fig. 2 Modèle éléments finis statique (a) - Directions de prolongement de $\sigma(b)$

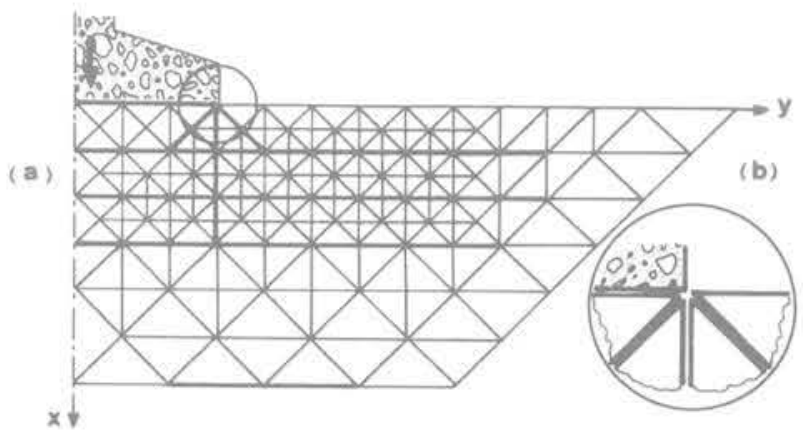

Fig. 3 Modèle éléments finis cinématique (a) - Discontinuités cinématiques (b)

(b)

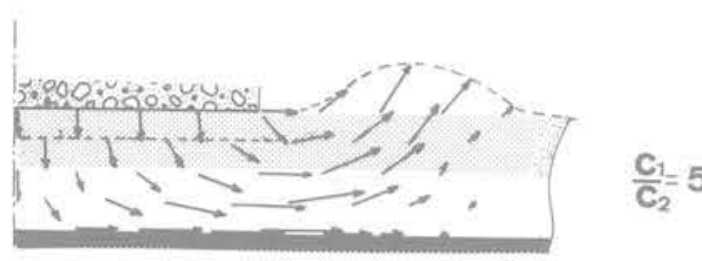

(a)

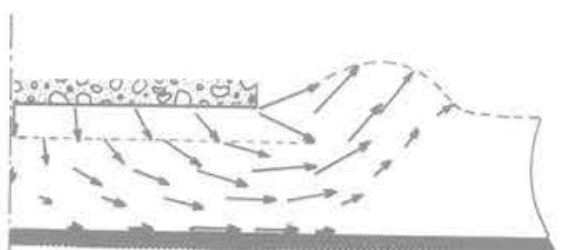

(c)

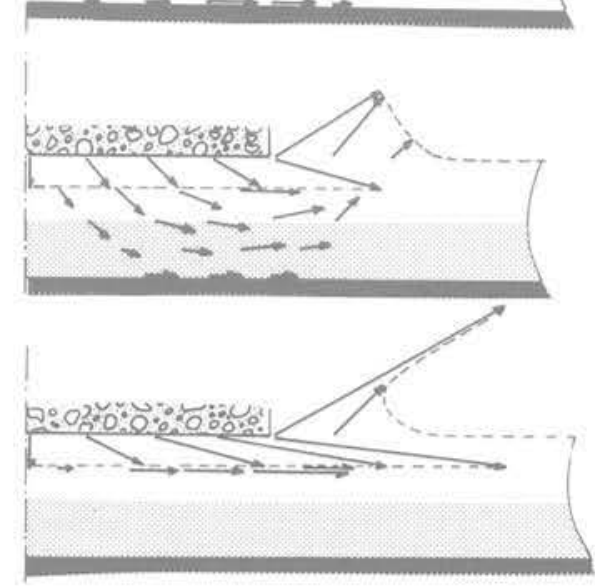

$\frac{c_{1}}{c_{2}}=1$

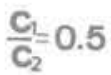

$\frac{C_{1}}{C_{2}}=0.2$ 
$\mathrm{m}$ : degré de linéarisation du critère;

$\left[U_{]}\right]$: discontinuité cinématique tangentielle moyenne sur le segment $\ell^{\text {d }}$.

\subsection{Modèles étudiés}

Nous avons sélectionné 5 épaisseurs relatives globales variant de 0,25 à 2 afin de mettre en évidence l'influence du substratum indéformable sur la capacité portante.

2.4.1 Étude détaillée du cas $\frac{\mathrm{h}}{\mathrm{b}}=0,25$

\subsubsection{Approche cinématique}

Les champs de vitesses présentés sur la figure 4 correspondent aux solutions optimales des problèmes (7) pour le modèle cinématique correspondant à $\frac{h}{B}=0,25$. Ils sont obtenus à partir de la solution du problème en milieu homogène selon un processus développé en [1].

a) Le cas homogène présente une cinématique d'ensemble régulière utilisant largement les discontinuités cinématiques autorisées sous la fondation et au voisinage de la singularité que constitue le bord $\mathrm{A}^{\prime}$. II conduit à une valeur de $N_{c}$ égale à 6,75 , la solution exacte [5] valant 6,22 .

b) Quand la couche supérieure durcit, il se produit un "effet de plaque " sous la fondation, accompagné d'un élargissement de la zone de rupture dans la couche inférieure.

c) Quand la couche la plus molle occupe la partie supérieure, la déformation est concentrée au voisinage du point $A$; la couche inférieure est moins sollicitée jusqu'à ne plus l'être du tout quand on atteint le rapport $\frac{C_{1}}{C_{2}}=0,20$ (d).
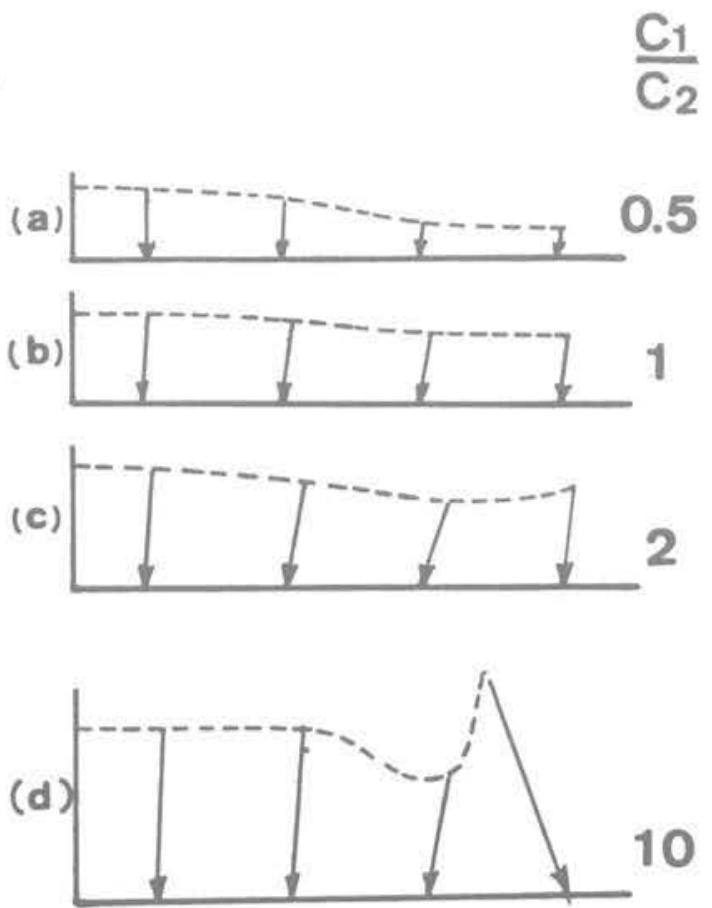

\subsubsection{Approche statique}

L'approche statique de ce cas a été effectuée par optimisations successives du problème (4). Sur ce modèle où l'interface sol-fondation est assez bien discrétisé, nous avons comparé les répartitions de contraintes suivant $\frac{C_{1}}{C_{2}}$ sur la figure 5 . On observe un affaiblissement de la contrainte normale au bord $A^{\prime}$ de la fondation quand la cohésion de la couche supérieure diminue (a) et un accroissement très net de cette contrainte ainsi qu'une inversion du sens de la contrainte tangentielle quand $\mathrm{C}_{1}$ devient très supérieur à $\mathrm{C}_{2}$ (d). Le champ $\underline{\sigma}$ optimum en milieu homogène conduit à $\mathrm{N}_{\mathrm{c}}=5,74$, valeur pratiquement symétrique de la borne cinématique 6,75 par rapport à la solution exacte ainsi approchée à $8 \%$ près. L'analyse des champs optimaux obtenus à partir de ce modèle entièrement discontinu confirme que la saturation du critère est observée essentiellement là où la cohésion est la plus faible (fig. $6 \mathrm{a}$ et $\mathrm{c}$ ) ou sous la base de la fondation (fig. $6 \mathrm{~b}$ ), chaque secteur angulaire noir indiquant que $\underline{g}$ vérifie $f\left(\sigma_{i j}\right)=0$ en ce sommet de triangle.

2.4.1.3 Comparaison des approches statique et cinématique

Si l'on représente la force $F^{(s)}$ obtenue à partir de (3) et la force $F^{(c)}$ obtenue à partir de $(6)$ et $(7)$ suivant $\frac{C_{1}}{C_{2}}$ on obtient la fourchette des valeurs encadrant $p_{\ell}$ exprimé sous la forme :

$$
P_{e}=C_{1} \times N_{c}\left(\frac{C_{1}}{C_{2}}, \frac{h_{1}}{h}, \frac{h}{B}\right)
$$

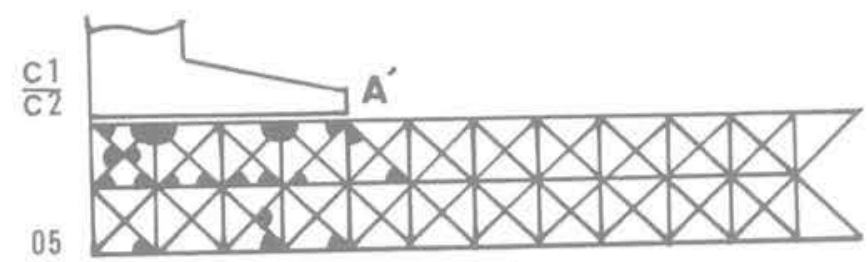

(a)

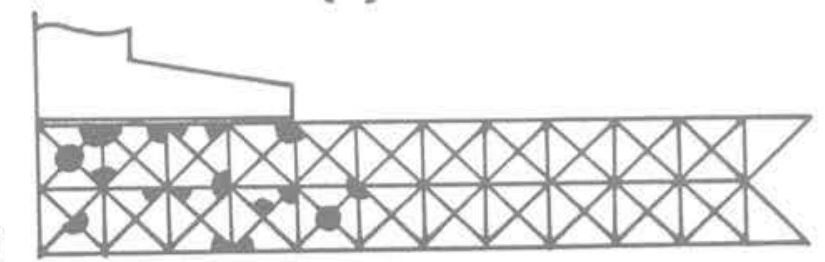

(b)

10

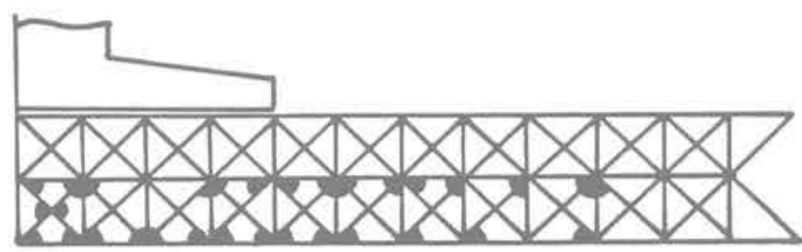

(c)

Fig. 6 Zones plastiques suivant $C_{1} / C_{2}$ 
La figure $7 \mathrm{~b}$ sur laquelle les valeurs exactes de $\mathrm{N}_{\mathrm{c}}$ sont indiquées par $\circ[5]$ montre que :

- L'écart entre les valeurs de $\mathrm{N}_{c}^{(\mathrm{s})}$ " statique" et $\mathrm{N}_{c}^{(\mathrm{c})}$ " cinématique " augmente au fur et à mesure que l'on s'éloigne du cas homogène.

- La solution statique varie peu en deçà de $\frac{C_{1}}{C_{2}}=0,50$ tandis que $\mathrm{N}_{\mathrm{c}}^{(c)}$ croît exagérément, le nombre d'éléments intervenant dans le calcul de la puissance dissipée étant alors insuffisant.

2.4.2 Étude du cas $\frac{h}{B}=0,50$

Dans le cas $\frac{h}{B}=0,50$ nous avons représenté la fourchette de $\mathrm{N}_{\mathrm{c}}$ obtenue pour différentes épaisseurs relatives de la couche supérieure (fig. 8 ) en indiquant dans la mesure du possible les valeurs fournies par la méthode des cercles de glissement [6] qui correspondent à un substratum situé à une profondeur infinie. Tant que l'épaisseur de la couche supérieure demeure faible (fig. $8 \mathrm{a}$ ) l'écart entre $\mathrm{N}_{c}^{(\mathrm{s})}$ et $\mathrm{N}_{c}^{(c)}$ reste du même ordre de grandeur que celui enregistré dans le cas homogène soit :

$N_{c}^{(s)}=5,00(-5 \%) \leqslant N_{c}$ exact $=5,25$

$$
\leqslant \mathrm{N}_{c}^{(c)}=5,73(+9 \%) .
$$

A partir de $\frac{\mathrm{C}_{1}}{\mathrm{C}_{2}}=0,50$ (fig. $8 \mathrm{c}$ ) la solution fournie par les cercles de glissement dans I'hypothèse d'une couche 2 infinie, conduit à des valeurs inférieures aux solutions statiques obtenues. Dans ce cas, la présence du substratum accroît fortement la capacité portante quand la couche dure est superficielle. L'écart relatif entre $N_{c}^{(s)}$ et $N_{c}^{(c)}$ atteint $30 \%$ pour $\frac{C_{1}}{C_{2}}=10$. Quand la couche superficielle est la plus molle, les points* correspondant aux cercles de glissement conduisent à surestimer notablement la capacité portante et ce d'autant plus que $\frac{h_{1}}{h}$ est faible (fig. $8 a$ ). On peut remarquer que l'allure des courbes "statiques " suit d'assez près l'allure de celle qui joindrait les points correspondant aux cercles de glissement.

L'abaque de la figure 9 montre l'évolution de $\mathrm{N}_{\mathrm{c}}$ suivant $\frac{C_{1}}{C_{2}}$ pour différents niveaux de l'intercouche. Les courbes ont été tracées en interpolant le coefficient $N_{c}$ de façon proportionnelle au rapport $\frac{N_{c}^{(s)}}{N_{c}^{(c)}}$ entre les courbes statique et cinématique de $N_{c}$ en milieu homogène.

\subsection{3 Étude du cas $\frac{h}{B}=1$}

En milieu homogène, le modèle cinématique de la figure 3 conduit à $\mathrm{N}_{c}^{(c)}=5,39$ à partir du champ de vitesses de la figure 10 . Tandis que le modèle statique de la figure 2 conduit à $\mathrm{N}_{c}^{(s)}=4,86$, soit :

$$
4,86(-5,4 \%) \leqslant N_{c} \text { exact }=5,14 \leqslant 5,39(+4,8 \%) \text {. }
$$

En milieu hétérogène, les bornes obtenues quand la couche superficielle est peu épaisse (fig. 11 a) sont très voisines et les valeurs cinématiques meilleures que celles fournies par les cercles de glissement. La solution exacte est approchée dans le pire des cas à
$11 \%$ près. L'écart entre $\mathrm{N}_{c}^{(s)}$ et $\mathrm{N}_{c}^{(c)}$ croît ensuite pour atteindre $22 \%$ dans le cas $\frac{h_{1}}{h}=0,50$ et $\frac{C_{1}}{C_{2}}=10$ (fig. $11 \mathrm{~b}$ ). Le champ de vitesses correspondant à une couche superficielle dure peu épaisse $\left(\frac{C_{1}}{C_{2}}=10, \frac{h_{1}}{h}=\right.$ $\left.\frac{1}{6}\right)$ de la figure 13 montre :

- un centre de rotation $\Omega$ situé à $\frac{B}{4}$ environ du bord $A^{\prime}$, - une translation verticale d'ensemble du sol voisin de la fondation,

- une amplification des vitesses dans la zone sous-jacente à la "plaque dure n et une remontée du sol quasi verticale au-delà de $\Omega$.

L'abaque de la figure 12 regroupe les résultats des interpolations du coefficient $\mathrm{N}_{\mathrm{o}}$ selon le principe défini en 2.4.2.

2.4.4 Étude du cas $\frac{h}{B}=1,50$

On observe globalement un comportement analogue à celui du cas précédent. Les valeurs de $N_{c}$ interpolées entre les deux approches sont regroupées sur la figure 14 .

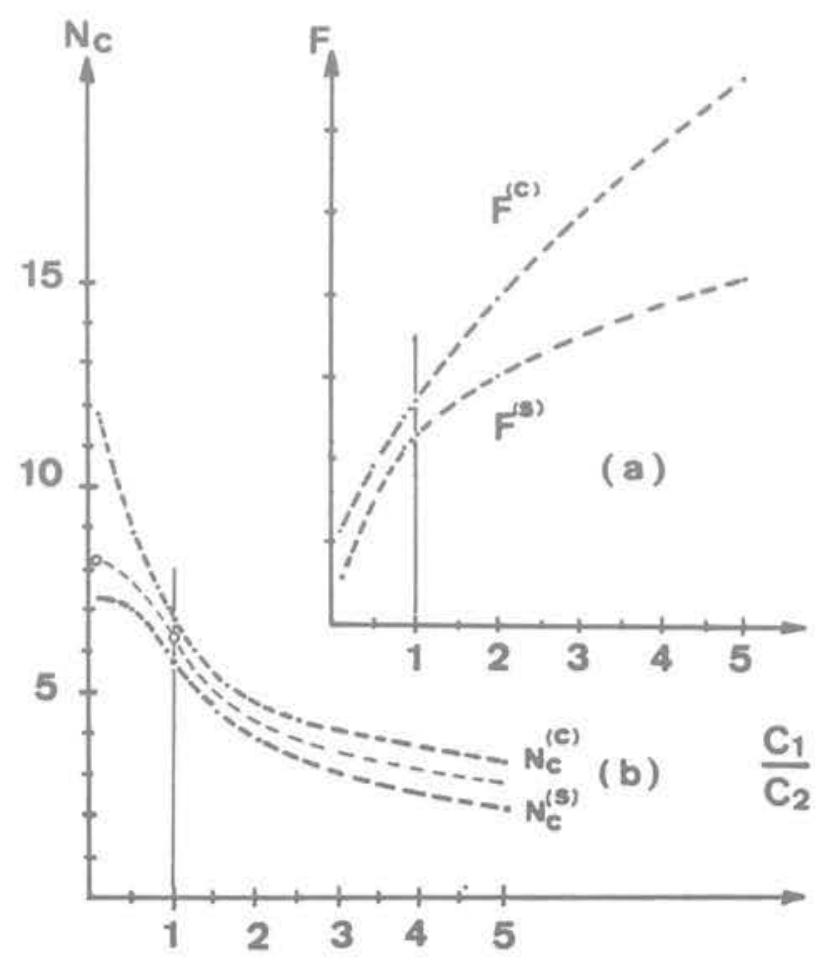

Fig. $7 N_{c}\left(C_{1} / C_{2}\right)$ pour $h / B=0,25$ 


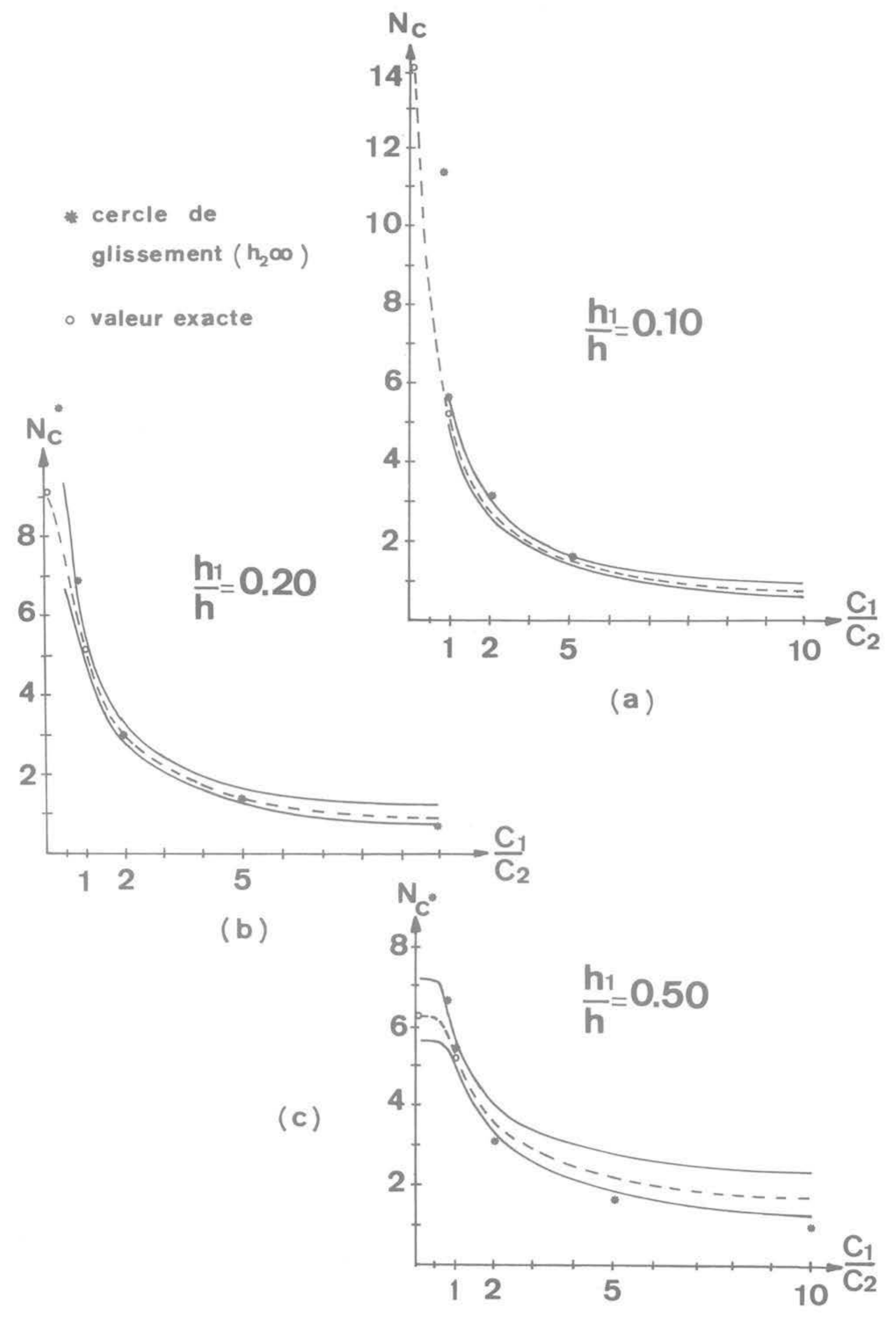

Fig. 8 Approches de $N_{c}$ pour $h / B=0,50$ 
2.4.5 Étude du cas $\frac{h}{B}=2$

Par rapport au cas $\frac{h}{B}=1$ de la figure 2, l'adjonction d'une zone complémentaire d'éléments permettant de situer le substratum à la cote $x=2 B$ entraîne une dégradation de la solution statique en milieu homogène qui passe de 4,86 à 4,73. La valeur fournie par le modèle cinématique $N_{c}^{(c)}=5,38$ rest très proche de la solution exacte $N_{c}=5,14$. L'abaque de synthèse pour les valeurs de $N_{c}$ interpolées est représenté sur la figure 15.

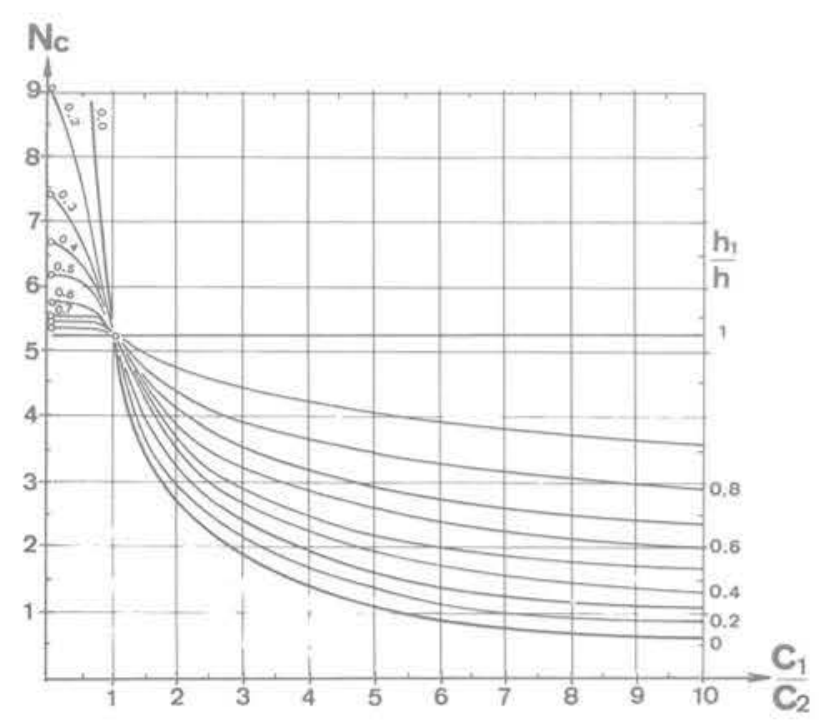

Fig. 9 Abaque $N_{c}\left(C_{1} / C_{2}, h_{1} / h\right)$ pour $h / B=0,50$
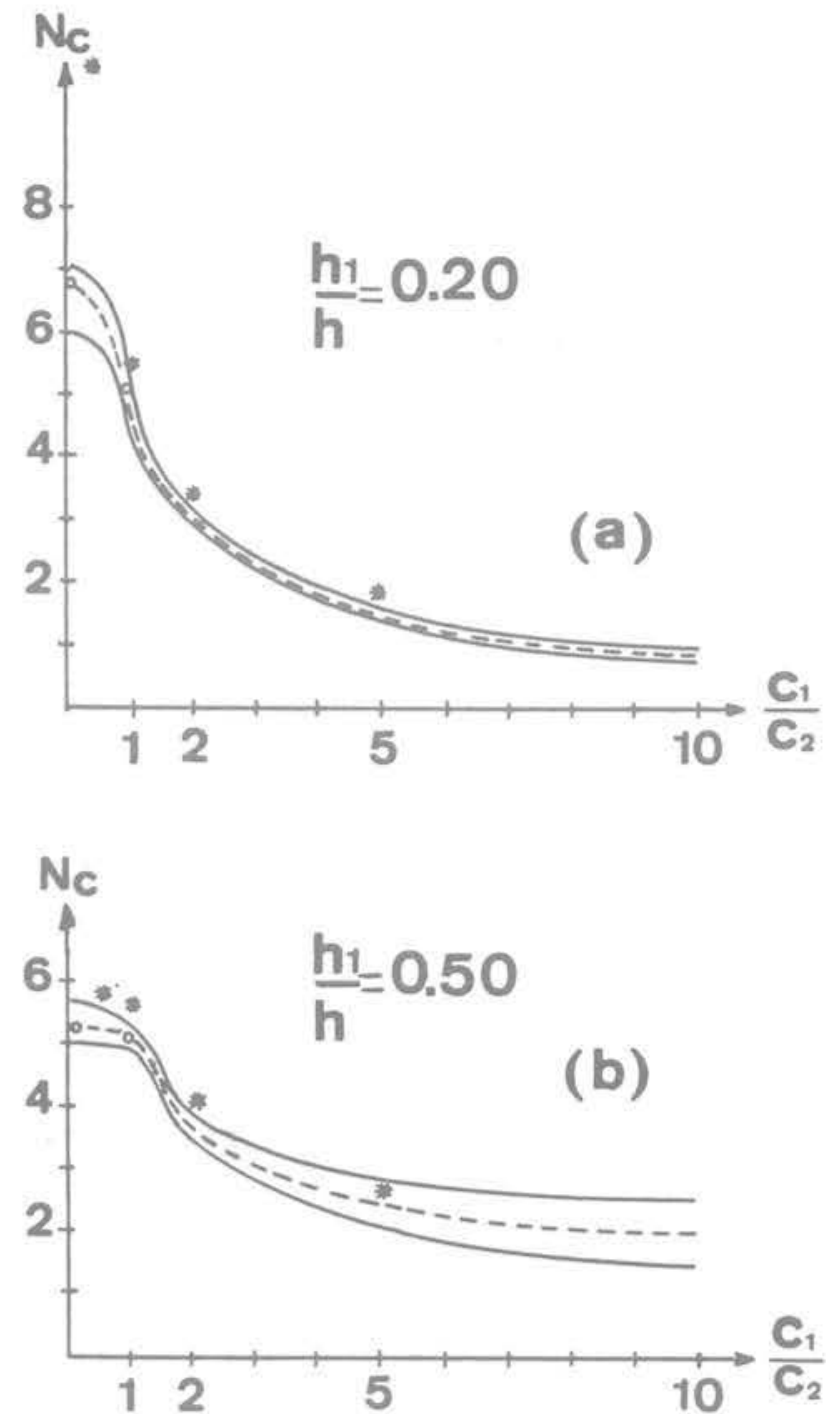

Fig. 11 Approches de $N_{c}$ pour $h / B=1$

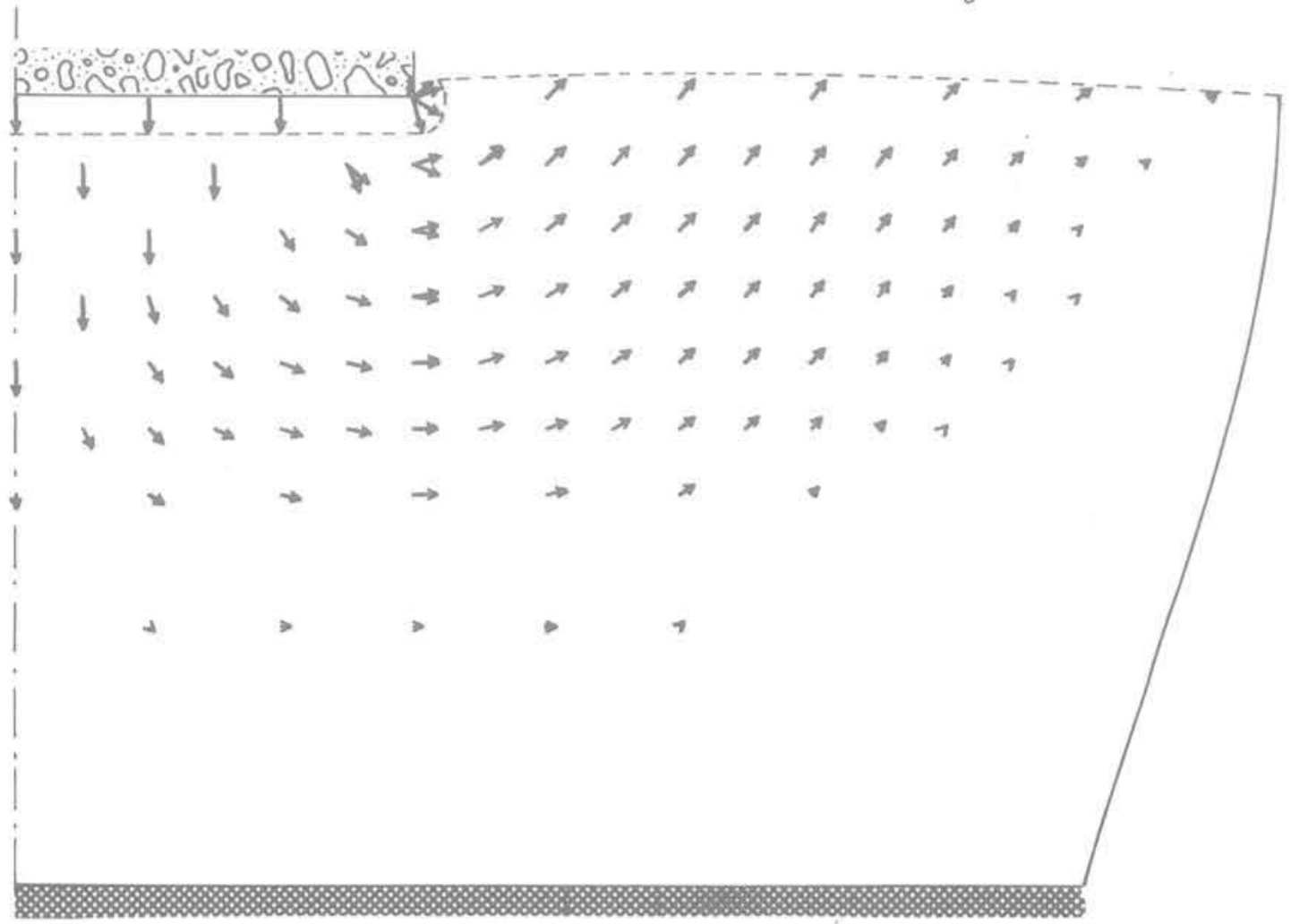

Fig. 10 Cinématique optimale pour $h / B=1$ en milieu homogène 


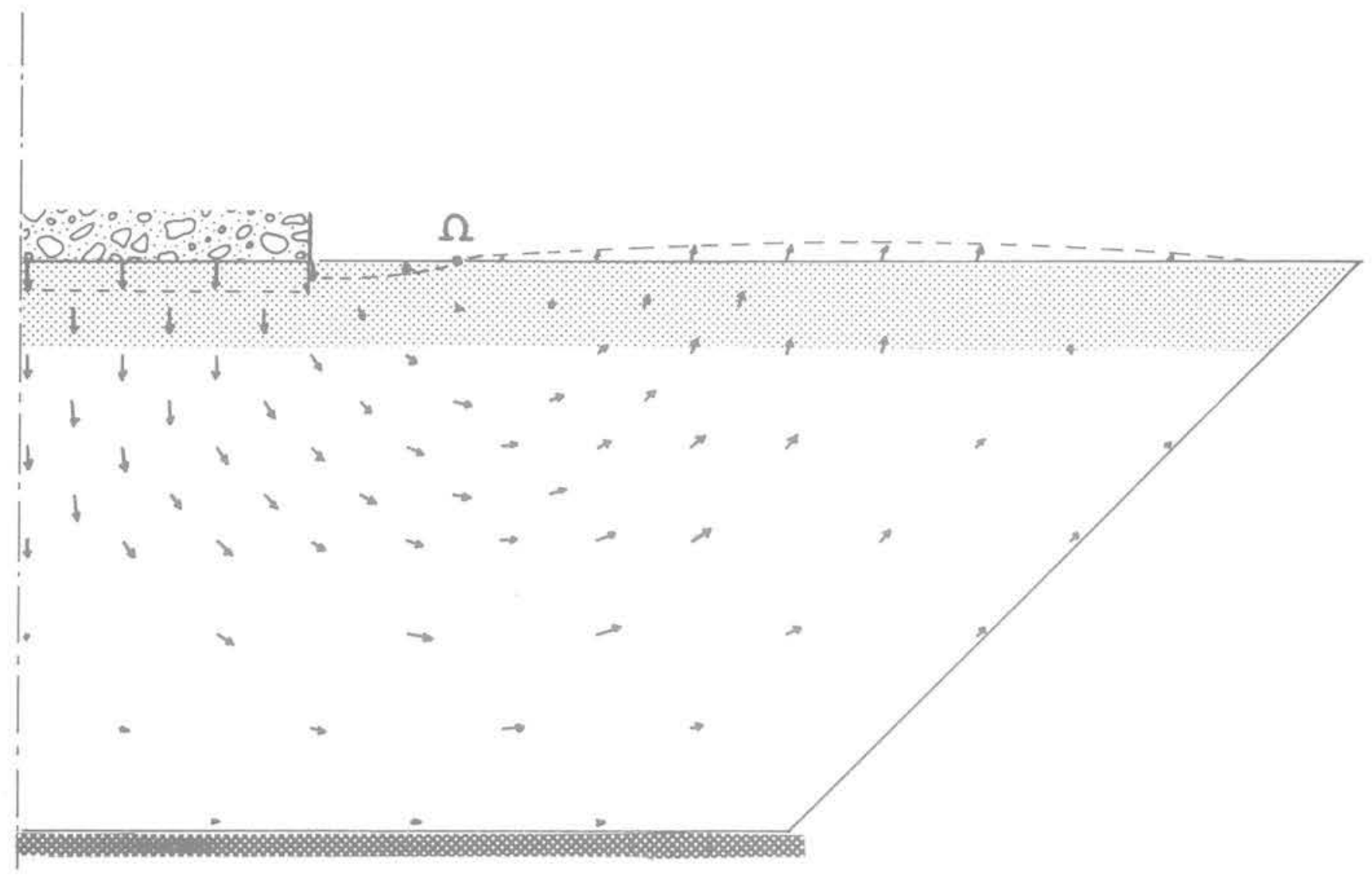

Fig. 12 Abaque $N_{c}\left(C_{1} / C_{2}, h_{1} / h\right)$ pour $h / B=1$
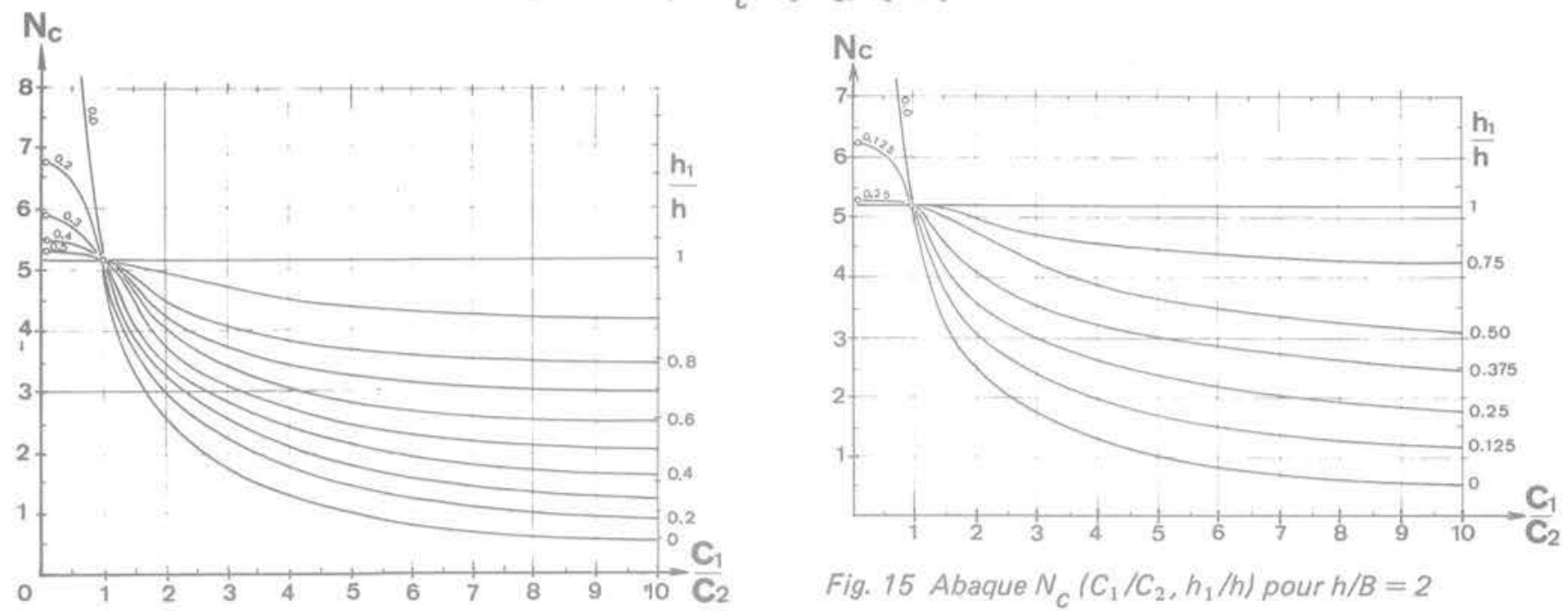

Fig. 15 Abaque $N_{C}\left(C_{1} / C_{2}, h_{1} / h\right)$ pour $h / B=2$

Fig. 13 Cinématique optimale pour $h / B=1$ avec $h_{1} / h=1 / 6$ et $C_{1} / C_{2}=10$

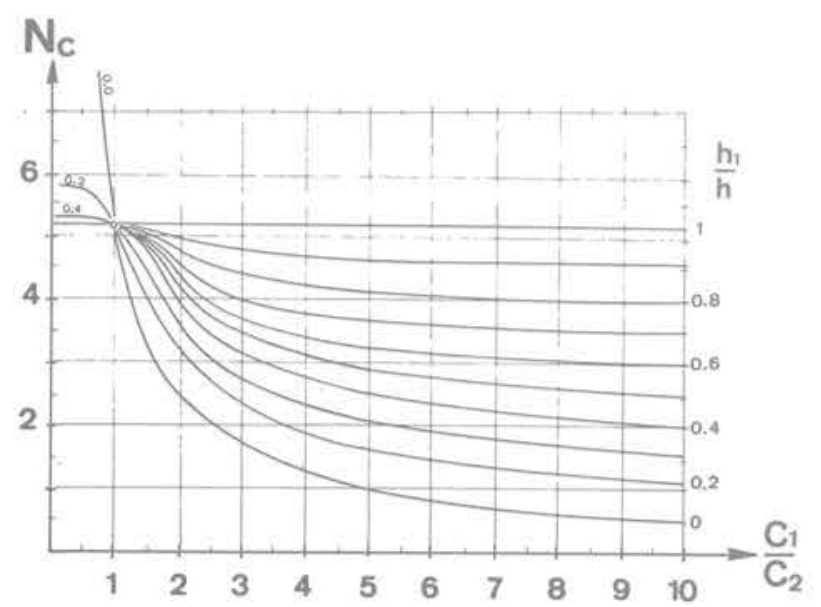

Fig. 14 Abaque $N_{C}\left(C_{1} / C_{2}, h_{1} / h\right)$ pour $h / B=1,50$

3 Approches statique et cinématique de la pression limite sous une fondation reposant sur une couche horizontale cohérente d'épaisseur $\mathbf{h}=\mathbf{B}$, présentant une inclusion hétérogène et limitée par un substratum indéformable

3.1 Conditions aux limites et hypothèses de calcul

La figure 16 présente les caractéristiques géométriques des problèmes abordés. Une inclusion d'épaisseur e de cohésion $\mathrm{C}_{2}$, située à une profondeur moyenne $h_{m}$, modifie la capacité portante d'une fondation de largeur $B$ reposant sur une couche d'épaisseur totale $\mathrm{h}=\mathrm{B}$ et de cohésion $\mathrm{C}_{1}$.

Le but des approches statique et cinématique est d'une part mesurer l'influence de l'épaisseur e et de la 


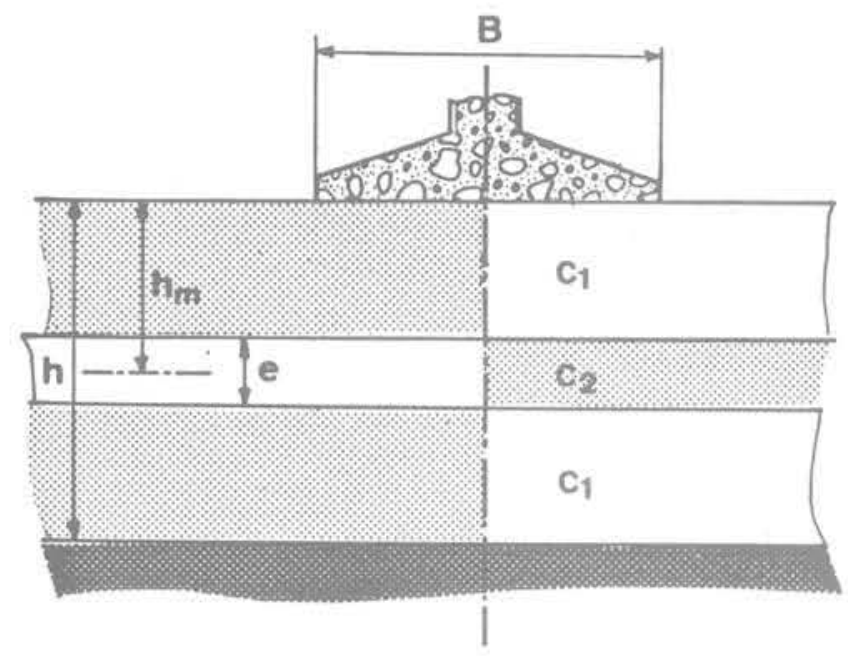

Fig. 16 Fondation sur couche homogène d'épaisseur $h=B$ avec inclusion

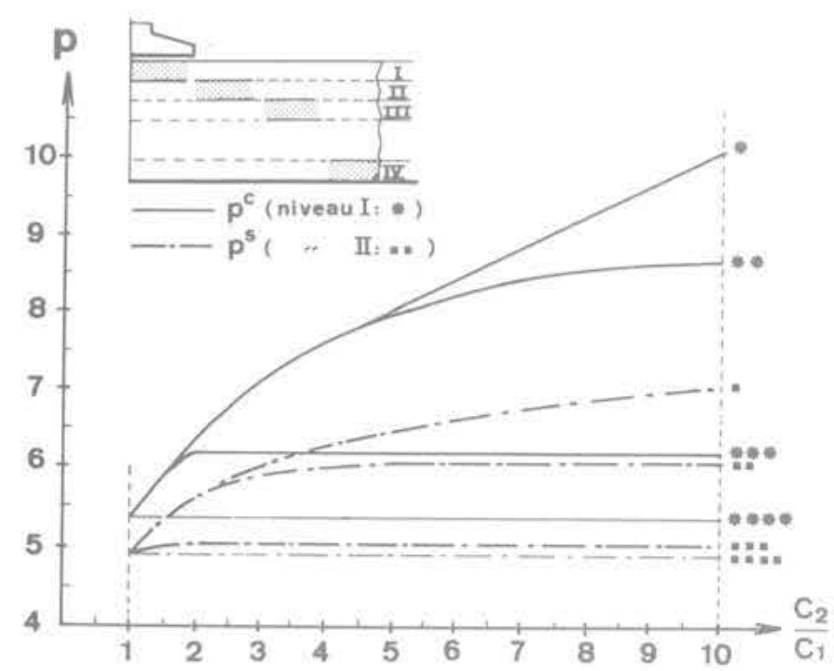

Fig. 17 Inclusion dure mince : approche de $p_{1}\left(C_{2} / C_{1}\right)$

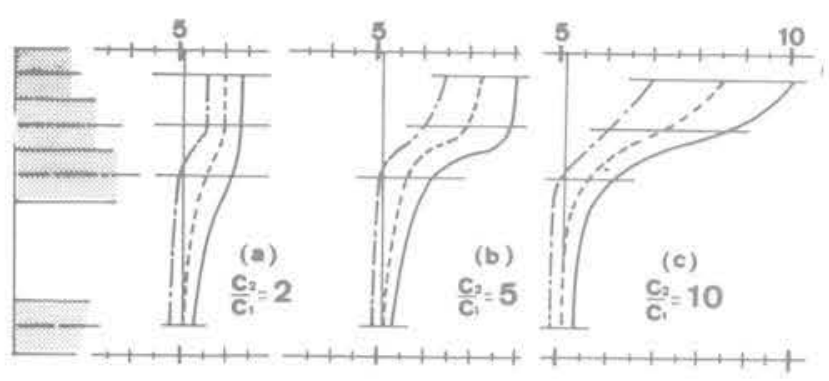

Fig. 18 Inclusion dure mince : approche de $p_{\text {, }}(\mathrm{hm}) /(\mathrm{h})$

position de l'inclusion sur les bornes de $p_{\ell}$ et d'autre part étudier le rapport $\frac{C_{1}}{C_{2}}$ critique à partir duquel le comportement du milieu reste insensible à l'augmentation de l'hétérogénéité mécanique.

\subsection{Modèles étudiés}

L'approche statique consiste à résoudre le problème (3) vu en $\mathbf{2 . 2}$ pour la fondation de la figure 16 sur l'ensemble des champs de contraintes linéaires licites décrits en $\mathbf{2 . 2}$ (voir fig. 2). L'approche cinématique revient à résoudre le problème (7) comme en 2.3. II convient de souligner que pour chaque approche tous les résultats sont obtenus à partir de la même discrétisation (fig. 2 et 3 ) et que la solution correspondant au milieu homogène sert de base de départ à la recherche de solutions de cas hétérogènes [1]. D'autre part les intercouches sont systématiquement des lignes de discontinuités en contraintes ou en vitesse.

\subsection{Inclusion dure}

Nous avons encadré la valeur de $p_{\ell}$ dans le cas $\frac{C_{1}}{C_{2}} \leqslant 1$, soit une inclusion dure d'épaisseur relative $\frac{\mathrm{e}}{\mathrm{h}}$ égale à $\frac{1}{6}$ puis $\frac{1}{3}$ et de position $h_{m}$ variable.

\subsection{1 Épaisseur relative $\frac{\mathrm{e}}{\mathrm{h}}=\frac{1}{6}\left(1 \leqslant \frac{\mathrm{C}_{2}}{\mathrm{C}_{1}} \leqslant 10\right)$}

La représentation graphique des résultats des approches effectuées à l'aide des modèles des figures 2 et 3 est indiquée sur la figure 17. Les fourchettes encadrant les valeurs les plus probables de $p_{\ell}$, interpolées en pointillés, suivant la position relative moyenne $\frac{\mathrm{hm}}{\mathrm{h}}$ de l'inclusion sont regroupées sur la figure 18 pour $\frac{C_{2}}{C_{1}}=2(a), 5(b)$ et 10 (c). On peut noter sur la figure 17 que les deux approches témoignent d'un comportement similaire. La présence de l'inclusion se fait sentir de façon croissante quand elle se rapproche de la fondation sauf pour les faibles hétérogénéités mécaniques pour lesquelles sa position influe peu sur $p$ (fig. 18a). Pour une position autre que la position superficielle, il existe une hétérogénéité mécanique $\frac{C_{2}}{C_{1}}$ au-delà de laquelle $\mathrm{p}$ ne varie plus. Cette limite est voisine de 8 quand $\frac{\mathrm{hm}}{\mathrm{h}}$ vaut 0,25 et voisine de 1,9 pour $\frac{h m}{h}=0,42$. La figure 17 montre que l'approche statique conduit plus rapidement à cette raideur limite que l'approche cinématique, confirmant ainsi la tendance vue en 2.4.1.

\subsection{2 Épaisseur relative $\frac{\mathrm{e}}{\mathrm{h}}=\frac{1}{3}\left(1 \leqslant \frac{\mathrm{C}_{2}}{\mathrm{C}_{1}} \leqslant 10\right)$}

Les figures 19 et 20 regroupent les résultats de l'évolution des approches de $p$ suivant le rapport $\frac{C_{2}}{C_{1}}$ et la position relative de l'inclusion. On peut souligner l'analogie de comportement avec les résultats concernant l'épaisseur relative $\frac{e}{h}=\frac{1}{6}$ excepté l'évolution de $p$ qui varie fortement suivant $\frac{h m}{h}$ à partir de $\frac{C_{2}}{C_{1}}=5$.

\subsection{Inclusion molle}

Nous avons étudié suivant les données de la figure 16 le cas complémentaire du précédent correspondant à la présence d'une inclusion molle d'épaisseur relative $\frac{\mathrm{e}}{\mathrm{h}}=\frac{1}{6}$ et $\frac{1}{3}$. 
3.4.1 Épaisseur relative $\frac{\mathrm{e}}{\mathrm{h}}=\frac{1}{6}\left(1 \leqslant \frac{\mathrm{C}_{1}}{\mathrm{C}_{2}} \leqslant 10\right)$

La figure 21 montre l'évolution de $p^{(s)}$ et de $p^{(c)}$ suivant $\frac{C_{1}}{C_{2}}$ dans le cas d'une inclusion molle de position variable et d'épaisseur relative $\frac{e}{h}=\frac{1}{6}$. La chute de portance du sol est d'autant plus marquée, par rapport au milieu homogène, que l'inclusion est plus proche de la surface. Pour les deux approches, la relation $p\left(\frac{C_{1}}{C_{2}}\right)$ est pratiquement linéaire quand l'inclusion est au fond; par contre, la pression limite reste pratiquement constante au-delà de $\frac{\mathrm{C}_{1}}{\mathrm{C}_{2}}=2$ dans le cas d'une inclusion

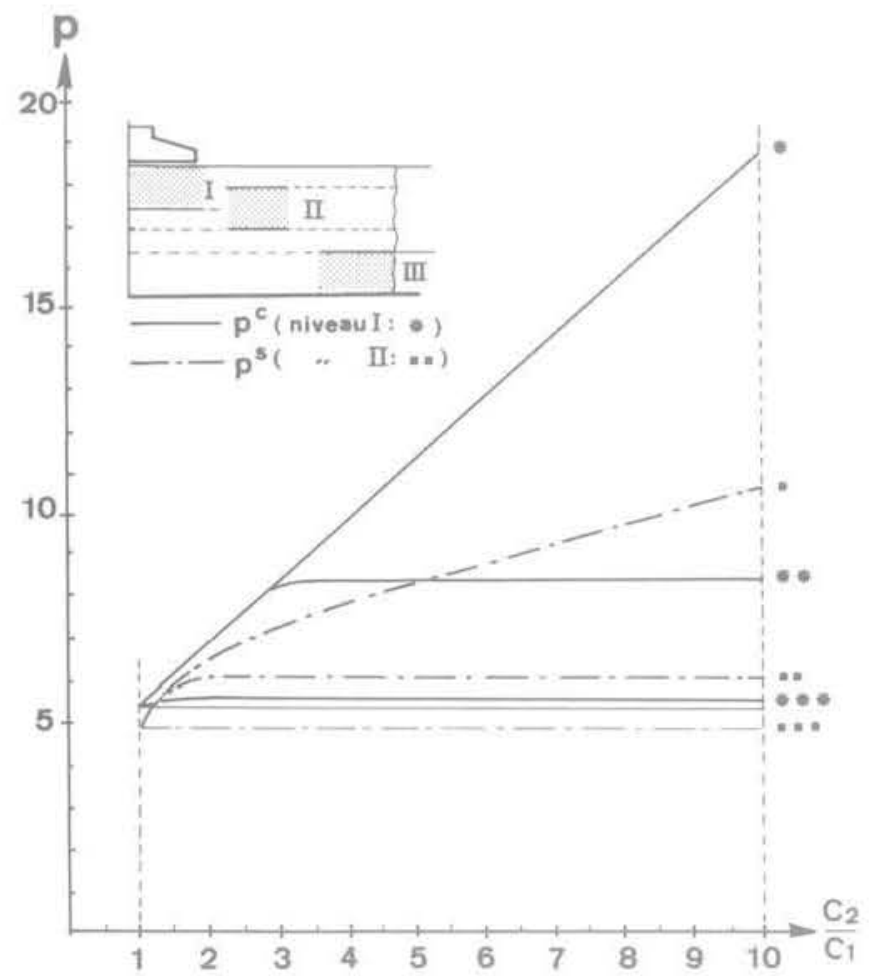

Fig. 19 Inclusion dure épaisse : approches de $p_{1}\left(C_{2} / C_{1}\right)$

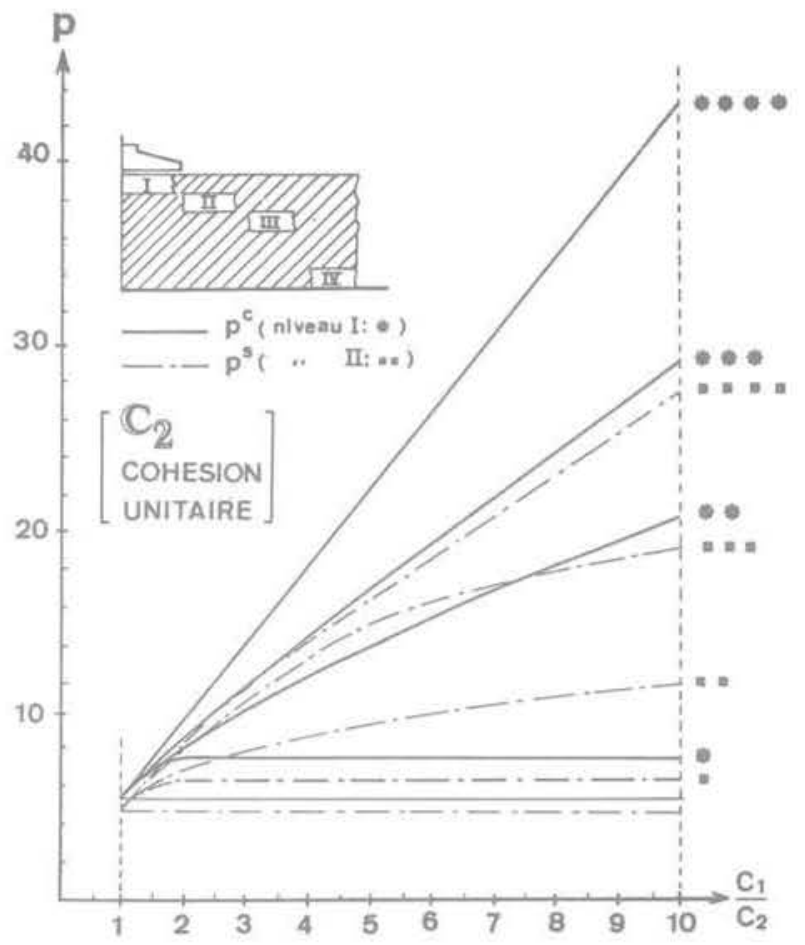

Fig. 21 Inclusion molle mince : approches de $p_{1}\left(C_{1} / C_{2}\right)$

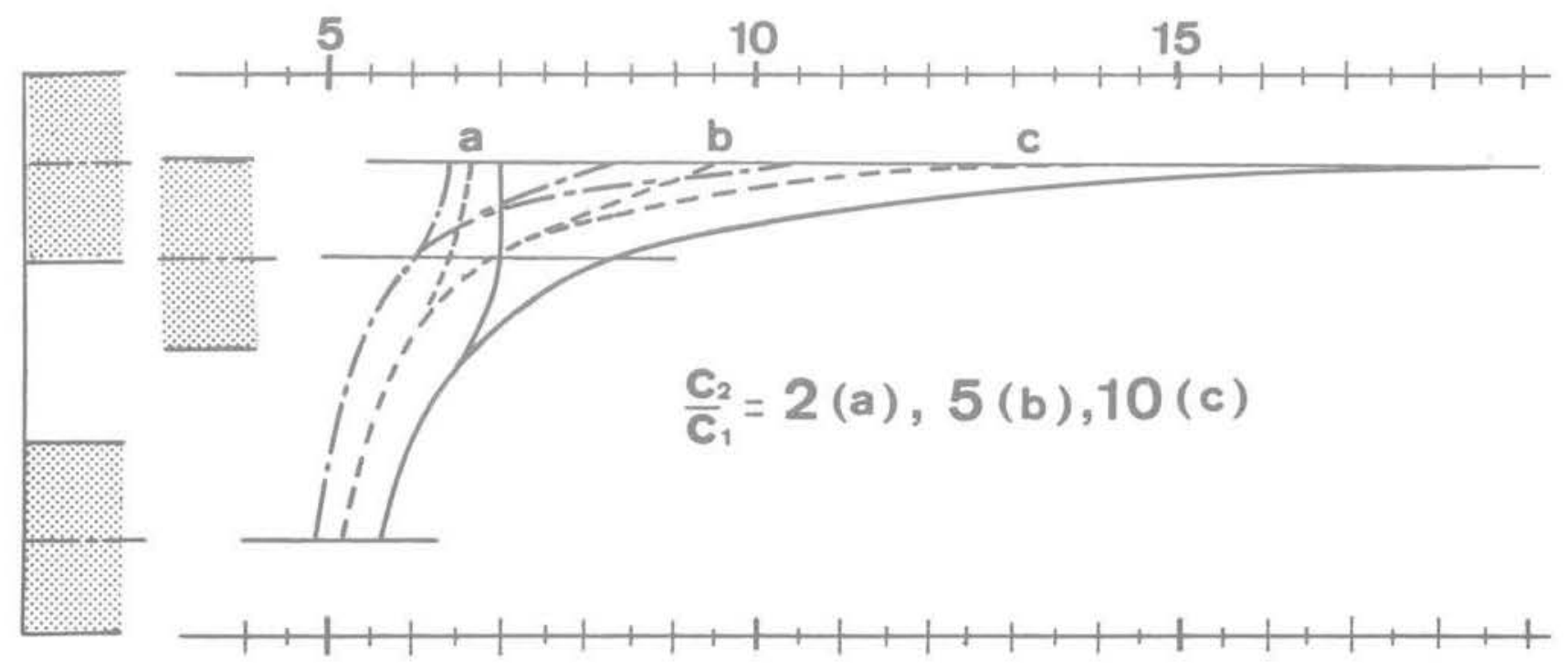

Fig. 20 Inclusion dure épaisse : approches de $p_{\text {f }}(\mathrm{hm} / \mathrm{h})$ 


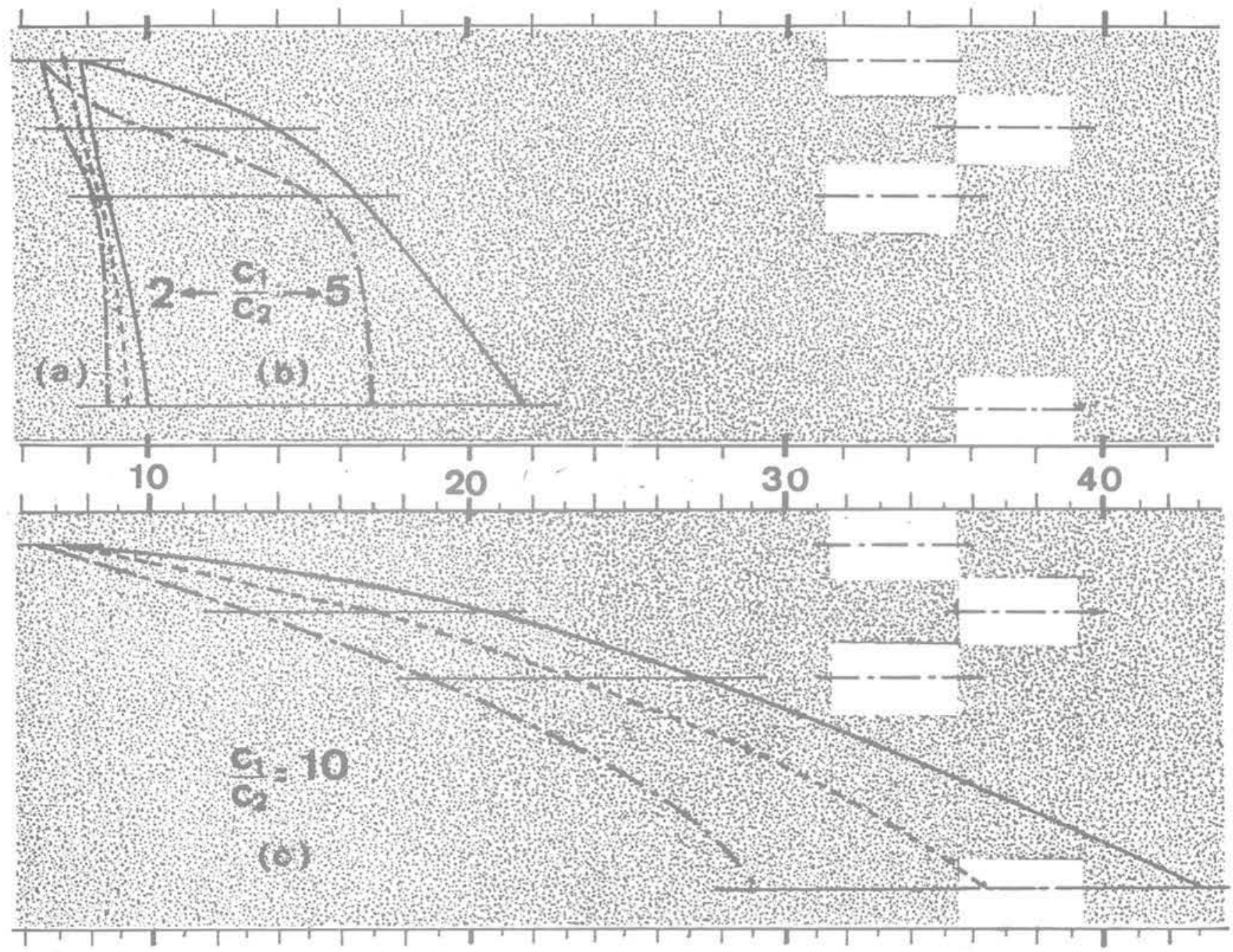

Fig. 22 Inclusion molle épaisse : approches de $p_{\text {, }}(\mathrm{hm} / \mathrm{h})$

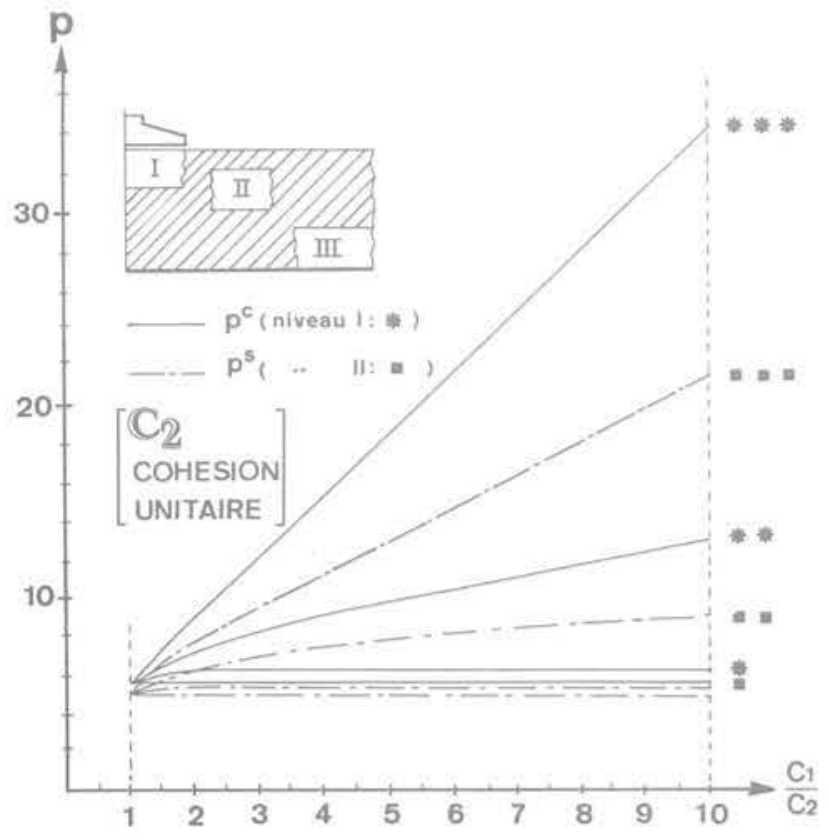

Fig. 23 Inclusion molle épaisse: approches de $p_{/}\left(C_{1} / C_{2}\right)$ de surface. La convergence ponctuelle observée entre les solutions statique et cinématique pour une position médiane de l'inclusion dans les cas $\frac{\mathrm{C}_{1}}{\mathrm{C}_{2}}=2$ et 5 (fig. $22 \mathrm{a}$ et b), provient probablement d'une cinématique particulièrement adaptée à ces cas d'hétérogénéité. Au-delà de cette position, la solution statique varie très peu alors que la solution cinématique continue de croître.

3.4.2 Inclusion molle $\frac{\mathrm{e}}{\mathrm{h}}=\frac{1}{3}\left(1 \leqslant \frac{\mathrm{C}_{1}}{\mathrm{C}_{2}} \leqslant 10\right)$

Les résultats des deux approches de $p_{\ell}$ sont indiqués sur la figure 23 sur laquelle on note que l'évolution des courbes $p^{(s)}$ et $p^{(c)}$ est très comparable et qu'elles sont pratiquement linéaires suivant le rapport d'hétérogénéité mécanique. Cette tendance se retrouve sur la figure 24 qui présente l'évolution des bornes statique et cinématique suivant la position de l'inclusion. La courbe intermédiaire en pointillés représente la valeur de $p_{\ell}$ interpolée entre $p^{(s)}$ et $p^{(c)}$ dans le même rapport que dans le cas homogène.

\subsection{Interprétation des cinématiques optimales}

\subsubsection{Inclusion dure « mince}

Pour une position de l'inclusion voisine de l'interface 


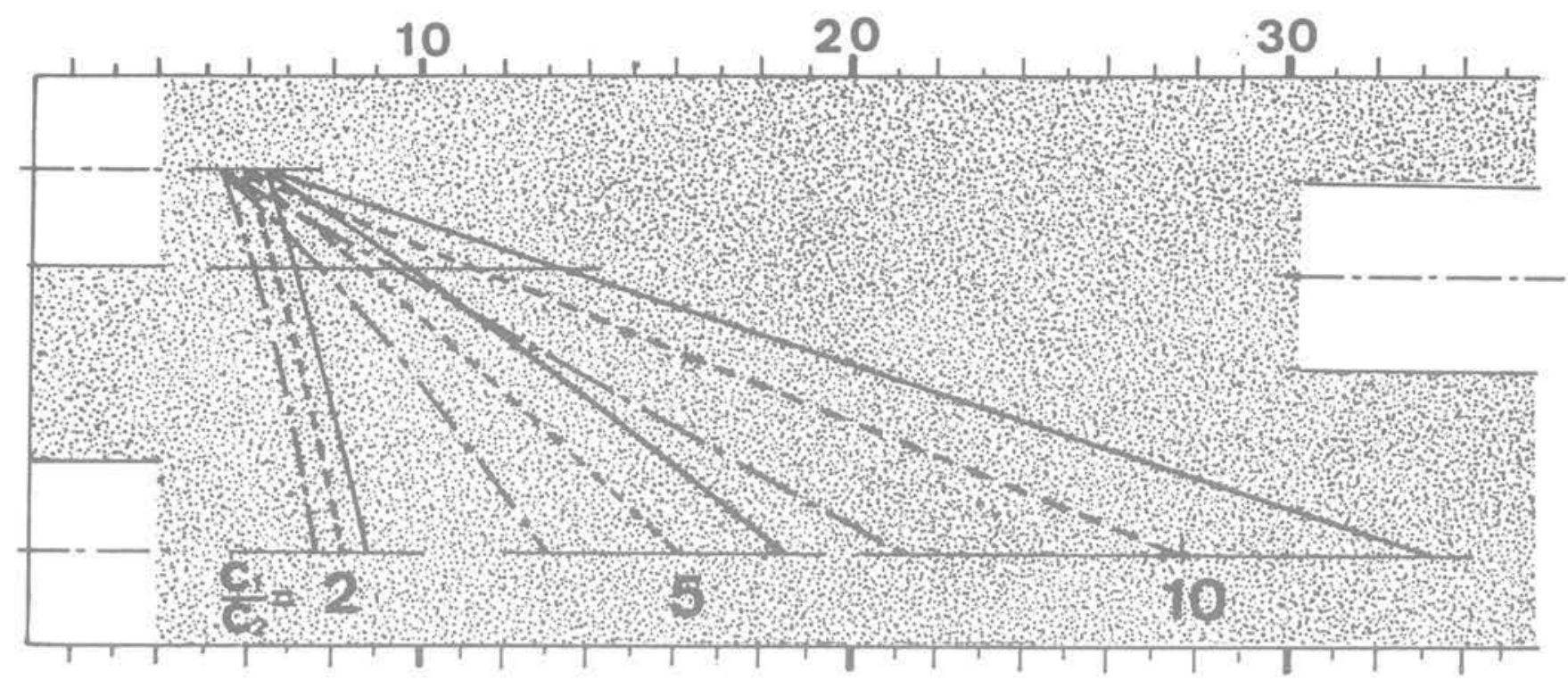

Fig. 24 Inclusion molle épaisse : approches de $p_{\text {, }}(\mathrm{hm}) /(\mathrm{h})$

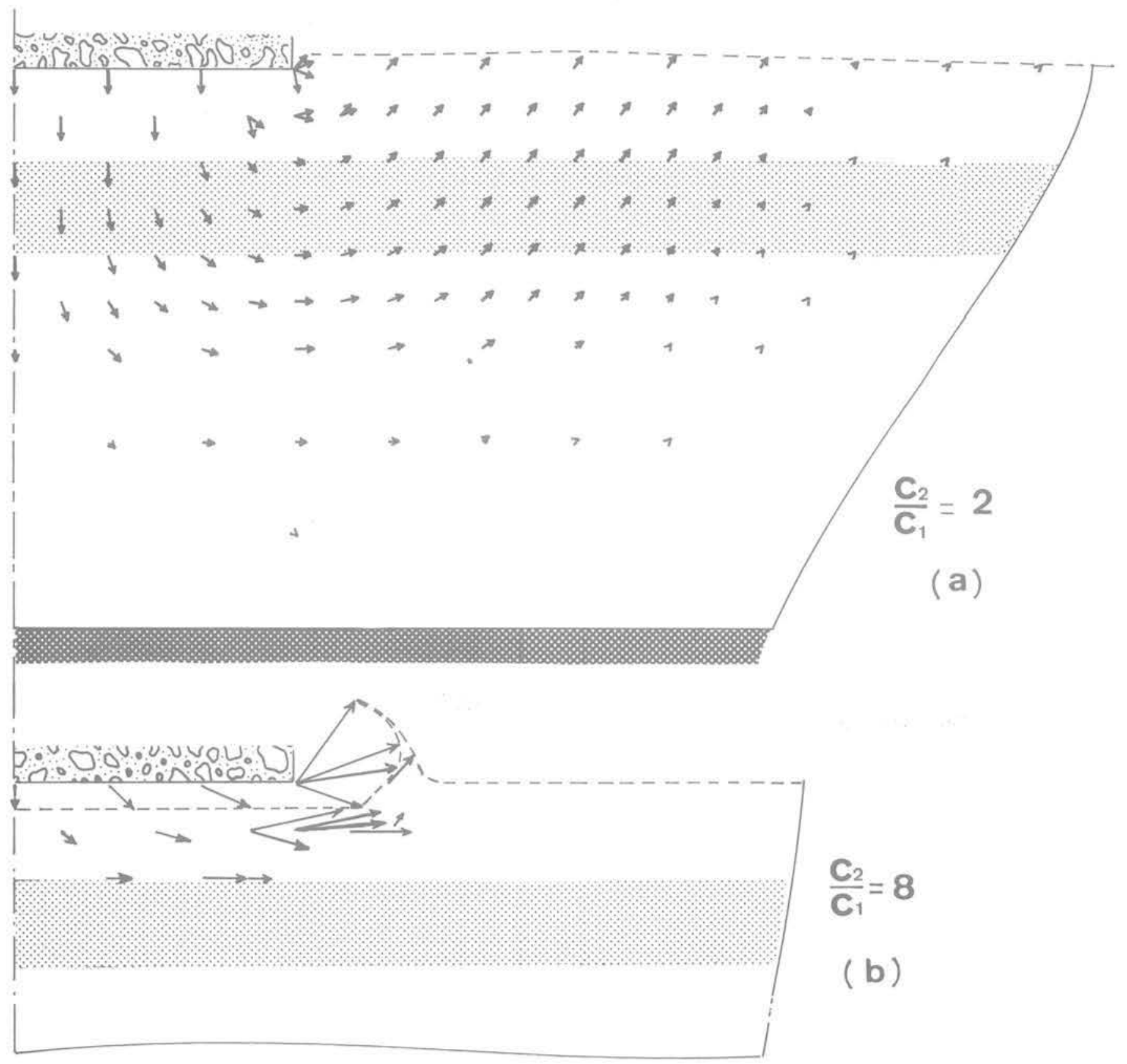

Fig. 25 Cinématiques optimales 


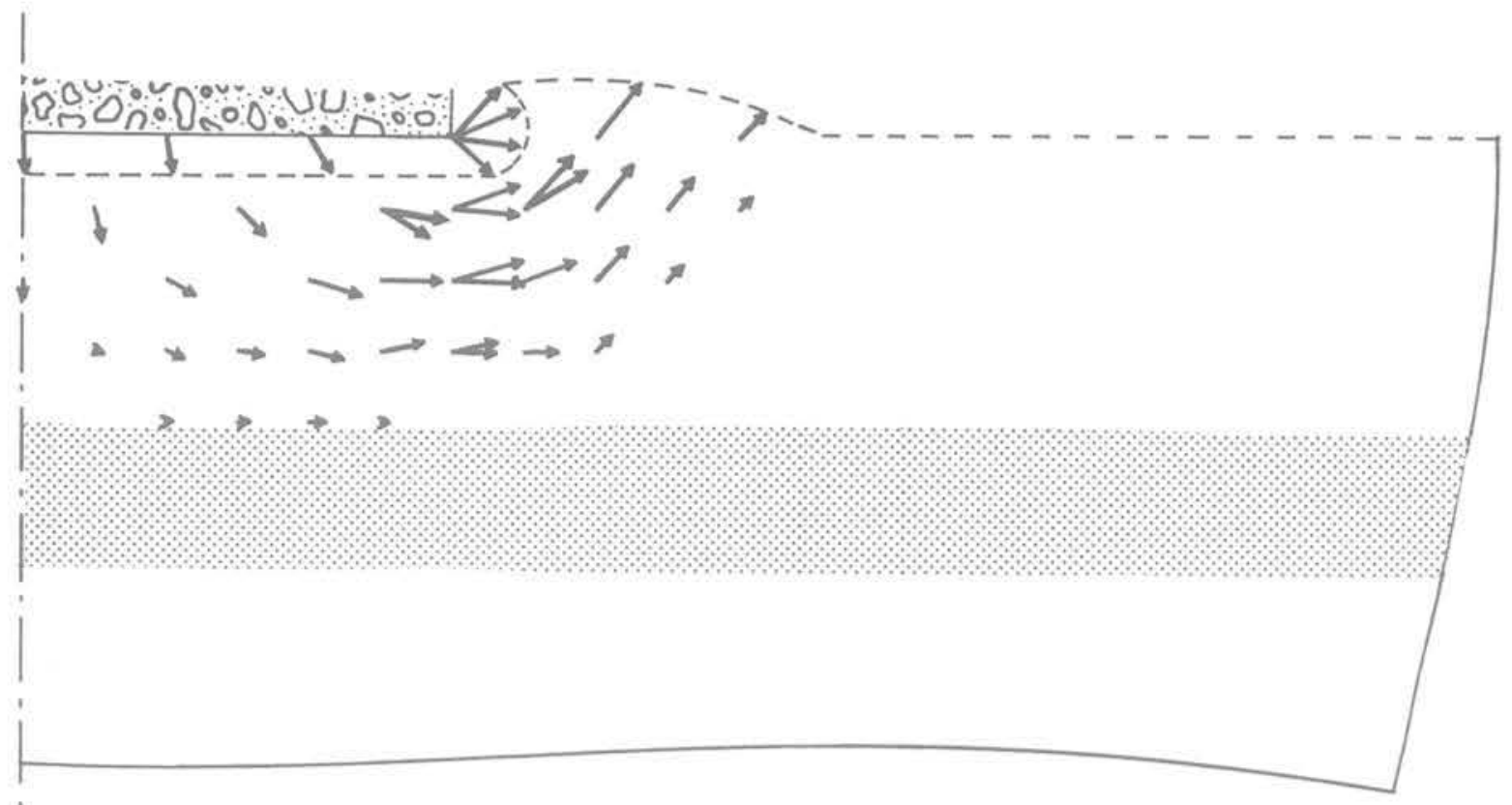

Fig. 26 Cinématique optimale : inclusion mince niveau III, $C_{2} / C_{1}=2$

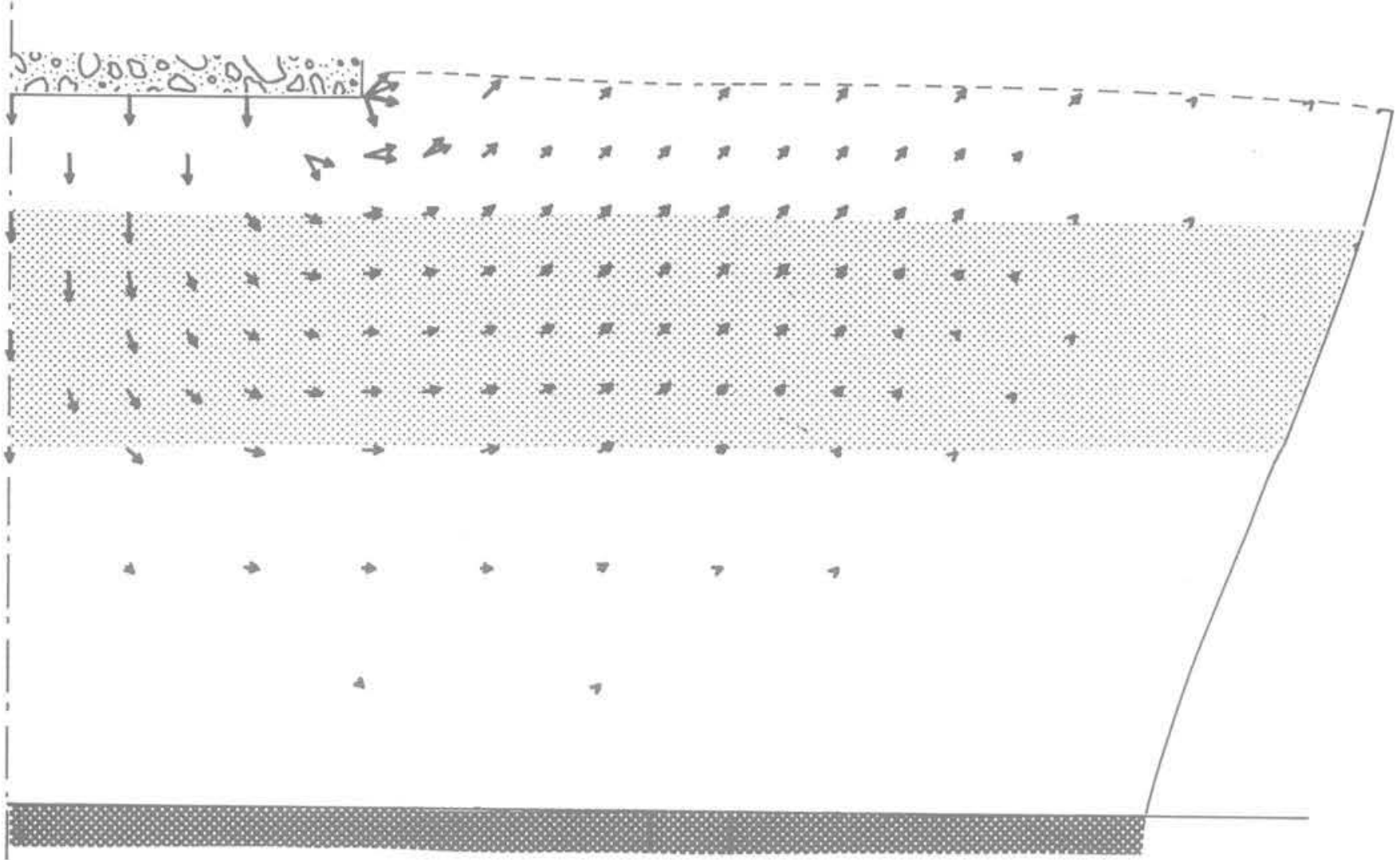

Fig. 27 Cinématique optimale : inclusion épaisse niveau II, $C_{2} / C_{1}=2$

sol/fondation et des valeurs modérées de $\frac{\mathrm{C}_{2}}{\mathrm{C}_{1}}$ les discontinuités cinématiques ne sont utilisées qu'au voisinage de la fondation (fig. 25 a), l'interface semellesol restant pratiquement collé.

Quand on atteint $\frac{\mathrm{C}_{2}}{\mathrm{C}_{1}}=8$, l'inclusion demeure rigide (fig. 25 b), seule la couche superficielle présente un mouvement très localisé au voisinage de la fondation : $50 \%$ de la puissance est dissipée sur l'interface sol-fondation et l'intercouche supérieure. Pour une position de l'inclusion voisine du milieu de la couche (fig. 26) on observe le même phénomène, l'inclusion devenant rigide pour une valeur de $\frac{C_{2}}{C_{1}}$ proche de 2 .
3.5.2 Inclusion dure "épaisse »

Le champ de vitesses est peu différent (fig. 27) de celui du milieu homogène (fig. 10) jusqu'à $\frac{C_{2}}{C_{1}}=3$, valeur pour laquelle on retrouve le comportement d'inclusion rigide de la figure $25 \mathrm{~b}$.

\subsubsection{Inclusion molle « mince »}

De globalement circulaire pour $\frac{\mathrm{C}_{1}}{\mathrm{C}_{2}}=2$ le mouvement évolue vers une translation horizontale de l'inclusion dès que l'on atteint $\frac{C_{1}}{C_{2}}=5$ quand cette dernière est 


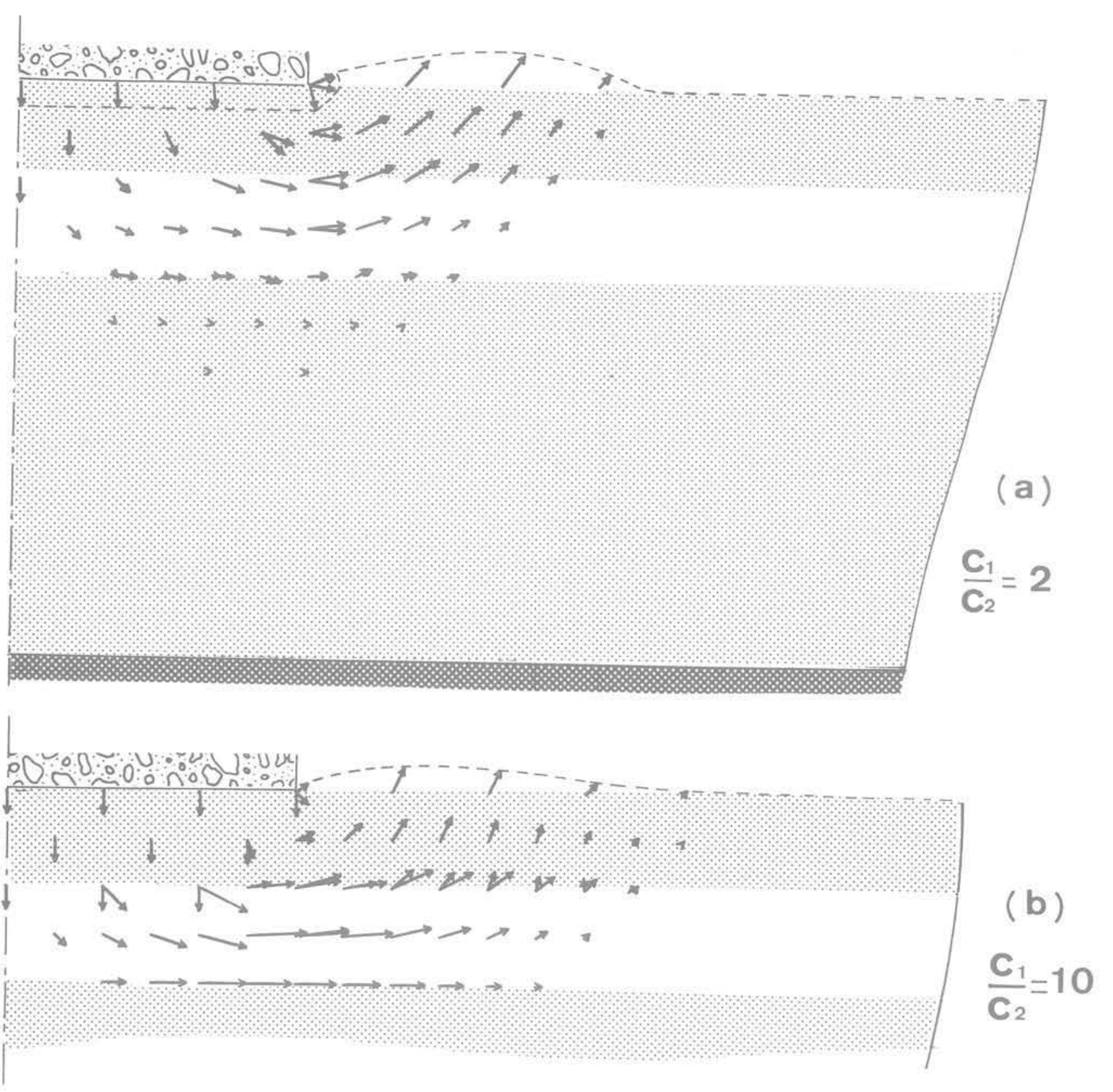

Fig. 28 Cinématiques optimales : inclusion mince molle, niveau II

voisine de la fondation (fig. 28). La couche de base demeure alors strictement rigide ainsi que le montre la figure $28 \mathrm{~b}$ pour $\frac{\mathrm{C}_{1}}{\mathrm{C}_{2}}=10$; la puissance dissipée par frottement atteint $20 \%$ de la puissance totale dissipée dans l'écoulement. Quand I'inclusion est située plus bas, le mouvement devient plus ample que dans le cas précédent et les discontinuités sont très peu utilisées tant que $\frac{\mathrm{C}_{1}}{\mathrm{C}_{2}}$ demeure faible (fig. 29a). Au contraire, on note un glissement très prononcé de l'inclusion par rapport à la couche inférieure qui demeure rigide pour $\frac{\mathrm{C}_{1}}{\mathrm{C}_{2}}=10$ (b)

\subsubsection{Inclusion molle «épaisse »}

Là encore, la couche inférieure qui repose sur le substratum demeure rigide (fig. 30 ). On retrouve les caractéristiques de "l'effet plaque " observé en 2.4.3: la couche dure demeure partiellement solidaire de la fondation et l'on peut mettre en évidence un centre de rotation $\Omega$ qui s'éloigne du bord de la semelle quand $\frac{C_{1}}{C_{2}}$ augmente $[(\mathrm{b})$ et (c)]. On notera l'évolution des discontinuités cinématiques, en particulier le glissement tangentiel vertical en A sur la figure $30 \mathrm{c}$. La puissance dissipée par frottement représente $28 \%$ de la puissance totale indépendamment de $\frac{C_{1}}{C_{2}}$.

\section{Conclusions}

La justification de cette étude numérique de la force portante est simple : les valeurs expérimentales dans le cas des matériaux cohérents sont en bon accord avec les résultats fournis par l'analyse limite quand la charge ultime expérimentale est clairement définie [4] [6]. La technique de la double approche dans un domaine où il n'existe pas de solution exacte associant un champ de contraintes et un champ de vitesses de déformation, nous paraît essentielle. L'approche cinématique seule est insuffisante au vu des écarts importants que l'on peut observer dans certains cas 

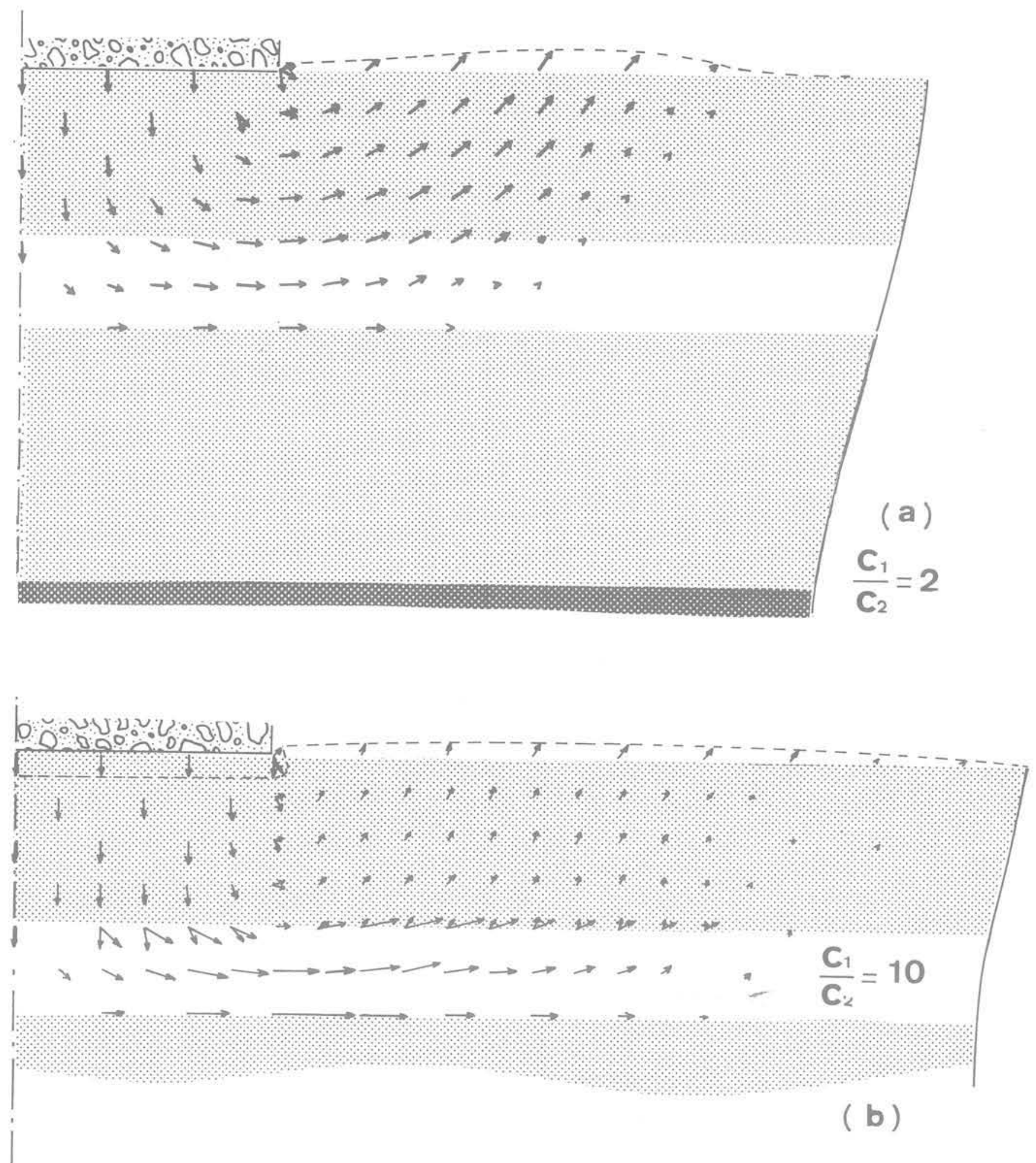

Fig. 29 Cinématiques optimales : inclusion mince molle, niveau II

comme nous l'avons souligné. La signification physique des schémas de rupture obtenus, dans lesquels les discontinuités cinématiques ne sont pas imposées mais seulement autorisées paraît rationnelle. Ils mettent en particulier en évidence un coefficient d'hétérogénéité mécanique critique dans le cas des inclusions du paragraphe 3 .

L'interpolation de $\mathrm{N}_{c}$ ou de $\mathrm{p}_{e}$ entre les bornes statique et cinématique est justifiée dans la mesure où les raisons de l'accroissement de l'écart observé suivant l'augmentation de l'hétérogénéité mécanique, semblent partagées entre les deux types d'approche:
- restriction des possibilités variationnelles des champs de vitesse de déformation et des champs de contraintes dans la partie inférieure du modèle (éléments cinématiques plus grossiers, éléments statiques semi-discontinus dans certains cas).

- présence d'une frontière rigide se traduisant par un accroissement de la puissance dissipée sur la frontière droite des modèles pour les rapports $\frac{C_{1}}{C_{2}}$ et $\frac{h_{1}}{h}$ élevés de l'étude du paragraphe 2 et nécessité d'imposer des conditions de prolongement des champs de contraintes, probablement trop proches de la fondation donc pénalisantes dans le cas de fortes hétérogénéités. 

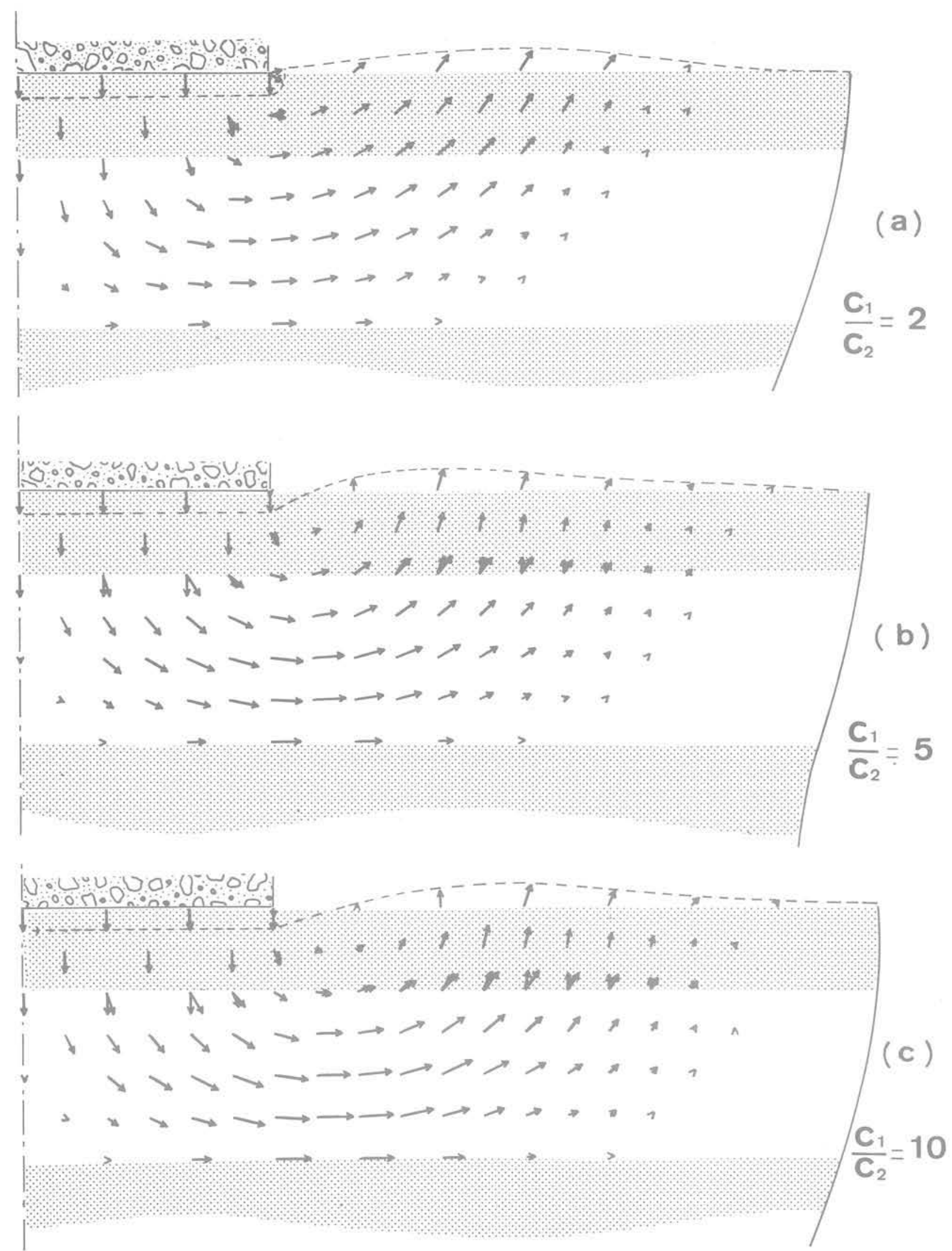

Fig. 30 Cinématiques optimales : inclusion molle épaisse

La technique retenue d'utiliser la même discrétisation pour traiter l'ensemble des cas hétérogènes pour une épaisseur relative donnée est séduisante vis-à-vis des possibilités offertes par les procédures de l'optimisation linéaire et seule compatible avec une exploration significative des paramètres d'hétérogénéité, néanmoins elle conduit à s'écarter parfois sensiblement de la discrétisation optimale.
Les procédés numériques utilisés ont été étendus aux problèmes présentant une symétries axiale de révolution [9] et récemment à l'étude des charges limites tridimensionnelles [2]. Ils devraient permettre une approche de la capacité portante de fondations circulaires et rectangulaires reposant sur des sols hétérogènes du type de ceux décrits dans la présente étude. 


\section{Références bibliographiques}

[1] A. Bottero (1981), "Contribution à l'étude du tassement et de la force portante des fondations superficielles reposant sur un sol multicouche limité par un substratum indéformable $»$. Thèse soutenue le 16 décembre 1981 pour obtenir le grade de Docteur ès-Sciences, Université de Grenoble.

[2] A. Bottero, J. Pastor, S. Turgeman (1980), "Calculs à la rupture par optimisation linéaire dans des modèles éléments finis tridimensionnels. $" 2^{\circ}$ Congrès international des Méthodes Numériques pour I'Ingénieur. Tome II, pp. 707-718.

[3] A. Bottero, R. Nègre, J. Pastor et S. Turgeman (1980), «Finite element method and limit analysis theory for soil mechanics problems» Computer Methods in Applied Mechanics and Engineering $n^{\circ} 22$ North Holland Publishing Cie pp. 131-149.

[4] A. Mahe, Y. Riou (1980), "Mise au point d'un modèle pour l'étude des milieux purement cohérents. " Journées Géotechniques I. N. S. A. Lyon 22-23 octobre 1980.

[5] J. Mandel et J. Salençon (1972), «Force portante d'un sol sur assise rigide (étude théorique). „ Géotechnique $22 n^{\circ} 1$, pp. 79-93.

[6] J. Obin (1972), «Force portante en déformation plane d'un sol verticalement non homogène. " Thèse présentée à l'Université de Grenoble, soutenue en février 1972 pour obtenir le grade de Docteur de Spécialité.

[7] J. Pastor (1978), «Analyse limite: détermination numérique de solutions statiques complètes. Application au talus vertical ". Journal de Mécanique Appliquée, vol. 2 pp. 167-196.

[8] J. Pastor, S. Turgeman (1976), "Mise en œuvre numérique des Méthodes de l'Analyse limite pour les matériaux de Von Mises et de Coulomb standard en déformation plane ». Mechanic Research Communication, $n^{\circ} 3$, pp. 469-474.

[9] J. Pastor, S. Turgeman (1979), «Formulation linéaire des méthodes de l'Analyse limite en symétrie axiale. "Communication au $4^{\circ}$ Congrès Français de Mécanique, Nancy.

[10] J. Salencon (1974), Théorie de la plasticité pour les applications à la mécanique des sols, Eyrolles, Paris. 